\title{
A Rigorous Derivation of Electromagnetic Self-force
}

\author{
Samuel E. Gralla, Abraham I. Harte, and Robert M. Wald \\ Enrico Fermi Institute and Department of Physics \\ University of Chicago \\ 5640 S. Ellis Avenue, \\ Chicago, IL 60637, USA
}

\begin{abstract}
During the past century, there has been considerable discussion and analysis of the motion of a point charge in an external electromagnetic field in special relativity, taking into account "self-force" effects due to the particle's own electromagnetic field. We analyze the issue of "particle motion" in classical electromagnetism in a rigorous and systematic way by considering a one-parameter family of solutions to the coupled Maxwell and matter equations corresponding to having a body whose charge-current density $J^{a}(\lambda)$ and stress-energy tensor $T_{a b}(\lambda)$ scale to zero size in an asymptotically self-similar manner about a worldline $\gamma$ as $\lambda \rightarrow 0$. In this limit, the charge, $q$, and total mass, $m$, of the body go to zero, and $q / m$ goes to a well defined limit. The Maxwell field $F_{a b}(\lambda)$ is assumed to be the retarded solution associated with $J^{a}(\lambda)$ plus a homogeneous solution (the "external field") that varies smoothly with $\lambda$. We prove that the worldline $\gamma$ must be a solution to the Lorentz force equations of motion in the external field $F_{a b}(\lambda=0)$. We then obtain self-force, dipole forces, and spin force as first order perturbative corrections to the center of mass motion of the body. We believe that this is the first rigorous derivation of the complete first order correction to Lorentz force motion. We also address the issue of obtaining a self-consistent perturbative equation of motion associated with our perturbative result, and argue that the self-force equations of motion that have previously been written down in conjunction with the "reduction of order" procedure should provide accurate equations of motion for a sufficiently small charged body with negligible dipole moments and spin. (There is no corresponding justification for the non-reduced-order equations.) We restrict consideration in this paper to classical electrodynamics in flat spacetime, but there should be no difficulty in extending our results to the motion of a charged body in an arbitrary globally hyperbolic curved spacetime.
\end{abstract}




\section{INTRODUCTION}

In classical electrodynamics in special relativity, Maxwell's equations

$$
\begin{aligned}
\nabla^{\nu} F_{\mu \nu} & =4 \pi J_{\mu} \\
\nabla_{[\mu} F_{\nu \rho]} & =0
\end{aligned}
$$

are most often considered in the context where the charge current source $J^{\nu}$ is a specified function of the spacetime coordinates $x^{\mu}=\left(t, x^{i}\right)$. Provided only that $J^{\nu}$ is conserved,

$$
\nabla_{\nu} J^{\nu}=0
$$

Maxwell's equations have a well posed initial value formulation. Furthermore, since Maxwell's equations are linear in $F_{\mu \nu}$, it makes perfectly good mathematical sense to allow $J^{\nu}$ be a distribution and to seek corresponding distributional solutions for $F_{\mu \nu}$. In particular, it makes perfectly good mathematical sense to consider point particle sources, i.e., distributional charge-current sources of the form (in global inertial coordinates ${ }^{1}$ )

$$
J^{\nu}=q u^{\nu} \delta^{(3)}\left(x^{i}-z^{i}(t)\right) \frac{d \tau}{d t}
$$

where $u^{\mu}$ is the unit tangent (i.e., 4 -velocity) of the worldline defined by $x^{i}(t)=z^{i}(t)$, and $\tau$ is the proper time along this worldline. Solutions to Maxwell's equations with an arbitrary point particle source of this form exist provided only that this worldline is timelike and that $q$ is constant along the worldline (as required by conservation of $J^{\nu}$, eq.(3i)) .

Another problem that is commonly considered in classical electrodynamics is the motion of a point particle in a given external field. Here one prescribes a Maxwell field $F_{\alpha \beta}^{\text {ext }}$ as a function of spacetime coordinates and one postulates the Lorentz force equation of motion for the point charge,

$$
m a_{\alpha}=q F_{\alpha \beta}^{\mathrm{ext}} u^{\beta}
$$

where $a^{\alpha} \equiv u^{\beta} \nabla_{\beta} u^{\alpha}$ is the 4-acceleration of the worldline. The Lorentz force equation is simply a system of second order ordinary differential equations for determining the worldline

\footnotetext{
${ }^{1}$ In arbitrary coordinates the right side would contain an additional factor of $(-g)^{-1 / 2}$ (see eq. (29) below).
} 
to which $u^{\alpha}$ is the unit tangent. It is a well posed equation that admits a unique solution for the motion of the particle for any specified $F_{\alpha \beta}^{\text {ext }}$ (which need not be a solution to Maxwell's equations) and any specified initial conditions of the particle.

Since Maxwell's equations are well posed for any specified point particle source and point particle motion is well posed in any specified Maxwell field, one might expect that the self-consistent coupled system, eqs.(11)-(2) and (4), together with

$$
m a_{\alpha}=q F_{\alpha \beta} u^{\beta}
$$

would also be well posed. (Here, eq.(6) differs from eq.(55) in that $F_{\alpha \beta}^{\text {ext }}$ has been replaced by the full Maxwell field $F_{\alpha \beta}$, which includes the contributions from the "self-field" of the point charge.) However, it is easily seen that this is not the case: The full Maxwell field $F_{\alpha \beta}$ is necessarily singular on the worldline of the particle, and, consequently, eq.(6) is ill-defined. Equations (11)-(21) and (4) together with eq.(6) simply do not make mathematical sense.

Nevertheless, some authors - most notably Dirac [1] — have attempted to proceed based on formal versions of these equations. However, in addition to being ad hoc, these treatments necessarily encounter mathematical and physical difficulties, such as the famous "infinite mass renormalization". In this picture, the infinite electromagnetic self-energy of a point charge is compensated by a negatively infinite material mass, rendering the combined (observable) mass finite. We see no justification for postulating that material mass could be negative (or infinite). Rather, as long as matter satisfies positive energy conditions, it is clear that a true point charge really would have an infinite total (observable) mass, and is therefore a physically unacceptable object. In short, the attempt to couple classical matter to electromagnetism via the point particle hypothesis (4) and the Lorentz force law (5) fails on both mathematical and physical grounds ${ }^{2}$.

Thus, it seems clear that the point particle charge-current (4) and Lorentz force law (5) cannot be fundamental. Rather, matter must be modeled as a continuum, with these equations (as well corrections, including self-force effects) emerging as an approximate description of the motion of "small bodies". In fact, much of the earliest work on the self-force problem [3, 4, 5] effectively followed this approach. However, a fundamental difficulty arises

\footnotetext{
${ }^{2}$ Similarly, in general relativity, Einstein's equation does not make mathematical sense for point mass sources [2]. Physically, matter would collapse to a black hole before a point mass limit could be achieved.
} 
in this approach. If one takes a limit to zero size in the straightforward way (i.e., at fixed charge and mass), one will simply recover the point particle description and its associated difficulties, such as infinite self-energy. But if one keeps the body at finite size, then all of the body's internal degrees of freedom will affect its motion, and the description of motion will depend in a complicated way on the details of the structure and initial state of the body.

This latter difficulty is commonly dealt with by postulating a rigid body, thereby eliminating the internal degrees of freedom. However, the notion of the "rigidity" of a body is incompatible with special relativity unless the body follows the orbits of a Killing field. Thus, rigidity places extremely restrictive a priori constraints on motion in special relativity and is an unacceptable starting point for a general analysis of the motion of a body. A proper continuum model must correctly allow for relativistic deformations. Mathematically, one should consider only models such that the self-consistent, coupled, Maxwell-charged-matter equations admit a well-posed initial value formulation, such as a charged fluid or a charged elastic solid (see [6] for further discussion).

However, our interest here is in deriving "universal" properties of the motion of small bodies that do not depend upon the details of any particular matter model. Thus, we wish to assume only the existence of a matter stress-energy tensor $T_{\mu \nu}^{M}$, which couples to the electromagnetic field via conservation of total stress-energy,

$$
\nabla^{\nu}\left(T_{\mu \nu}^{M}+T_{\mu \nu}^{E M}\right)=0
$$

where the electromagnetic stress-energy tensor is given by

$$
T_{\mu \nu}^{E M}=\frac{1}{4 \pi}\left(F_{\mu \alpha} F_{\nu}{ }^{\alpha}-\frac{1}{4} g_{\mu \nu} F_{\alpha \beta} F^{\alpha \beta}\right) .
$$

We will refer to the coupled system of eqs.(17) and (17) as the Maxwell and matter equations. All realistic bodies composed of continuum matter and charge-currents should satisfy the Maxwell and matter equations, and our results will be derived solely from these equations. ${ }^{3}$ Note that the Maxwell and matter equations are nonlinear in the electromagnetic field.

\footnotetext{
${ }^{3}$ These equations were also used in [7] as the starting point in the self-force context. Very recently, this work was improved [8] in a non-perturbative framework that provides a complete formalism for describing the motion of extended bodies, including self-field effects.
} 
Since the motion of a finite-size body will depend on its detailed composition as well as on the details of its internal states of motion, we must consider a limit where the body is shrunk down to zero size in order to have a chance at obtaining a simple, universal description of its motion. As mentioned above, normally such a limit is taken at fixed charge and mass, thereby reproducing the same difficulties as arise if one attempts to work directly with point particles. The main new idea in the present work is to consider a mathematically precise limit - based on suitable one-parameter families of exact solutions to the Maxwell and matter equations - that will enable us to avoid these difficulties and thereby to derive self-force effects in a mathematically rigorous manner. Following the basic ideas of [9] (which were formulated in the context of general relativity), we consider a modified point particle limit, wherein not only the size of the body goes to zero, but its charge and mass also go to zero. More precisely, we will consider a limit where, asymptotically, only the overall scale of the body changes, so, in particular, all quantities scale by their naive dimension. In this limit, the body itself completely "disappears", and its electromagnetic self-energy goes to zero. We will show in section III that the worldline, $\gamma$, to which the body shrinks down must be a solution to the Lorentz force equations of motion. (As we shall also see in section III, the electromagnetic self-energy of the body makes a finite, non-zero contribution to the mass, $m$, that appears in the Lorentz force equation.) Self-force effects (as well as dipole force and spin force effects) then arise as a first order perturbative correction to the center of mass motion of the body. We emphasize that our approach removes from any fundamental status both the point charge source (44) and the Lorentz force law (5). Rather, the notion of a "point charge" and the Lorentz force equation that it satisfies will be derived as a lowest order description of the electromagnetic field and bulk motion of a small (but extended) body $^{4}$ whose exact evolution is governed by the Maxwell and matter equations (1) 3 ), and (7).

Our derivation will enable us greatly clarify what is perhaps the most famous difficulty of the electromagnetic self-force problem: "runaway solutions". The output of the classic Abraham [3], Lorentz [4] and Dirac 1] analyses (as well as of many other analyses) is the

\footnotetext{
${ }^{4}$ Of course, the body of most interest to experimental phenomena, the electron, is an intrinsically quantummechanical object. Our results will apply to electrons only to the extent that a classical description of the motion of an electron can be justified.
} 
following equation:

$$
m a_{\alpha}=q F_{\alpha \beta}^{\mathrm{ext}} u^{\beta}+\frac{2}{3} q^{2}\left(g_{\alpha \beta}+u_{\alpha} u_{\beta}\right) \dot{a}^{\beta}
$$

usually known as the Abraham-Lorentz-Dirac (ALD) equation. The second term on the right side of this equation corresponds to the "self-force" on the charge resulting from its own electromagnetic field. This term makes the ALD equation third-order in time, rendering the status of its initial value formulation - and consequently its role in predicting actual particle motion - at best unclear. Furthermore, eq. (91) has solutions whose acceleration grows exponentially in time-behavior that is not observed in nature. Clearly, eq. (9) is disastrously pathological as it stands.

Historically, there have been three basic responses to these problems. The first response views eq. (9) to be a genuine prediction of classical particle electrodynamics, and seeks a resolution of the runaway difficulties by a treatment of the problem within the framework of quantum mechanics or quantum electrodynamics [10]. If so, then classical electrodynamics would be seriously deficient. The second response [1] holds that eq. (9) may be salvaged by introducing a set of rules designed to eliminate runaway behavior [1]. However, such rules are necessarily acausal in nature and would appear to be incompatible with the underlying causal behavior of classical electrodynamics. The third response maintains that the equation should be modified by the "reduction of order" procedure [11]. This procedure is based on the observation that eq. (9) is only expected to be valid to order $q^{2}$. Therefore, if we replace the $\dot{a}^{\beta}$ term in eq. (9) by the value it would take for Lorentz force motion in the background field $F_{\alpha \beta}^{\text {ext }}$, we should get an equation that is "equally valid" to order $q^{2}$. Thus, to order $q^{2}$, we may replace $a^{\alpha}$ on the right side of eq. (9) by

$$
a_{\alpha}=\frac{q}{m} F_{\alpha \beta}^{\mathrm{ext}} u^{\beta}
$$

and we may replace $\dot{a}^{\alpha}$ by

$$
\begin{aligned}
\dot{a}_{\beta} & =u^{\gamma} \nabla_{\gamma}\left(\frac{q}{m} F_{\beta \sigma}^{\mathrm{ext}} u^{\sigma}\right) \\
& =u^{\sigma} u^{\gamma} \nabla_{\gamma}\left(\frac{q}{m} F_{\beta \sigma}^{\mathrm{ext}}\right)+\left(\frac{q}{m} F_{\beta \sigma}^{\mathrm{ext}}\right) \frac{q}{m} F_{\delta}^{\mathrm{ext} \sigma} u^{\delta}
\end{aligned}
$$


The resulting equation

$$
m a_{\alpha}=q F_{\alpha \beta}^{\mathrm{ext}} u^{\beta}+\frac{2}{3} q^{2}\left(g_{\alpha}{ }^{\beta}+u_{\alpha} u^{\beta}\right)\left[u^{\sigma} u^{\gamma} \nabla_{\gamma}\left(\frac{q}{m} F_{\beta \sigma}^{\mathrm{ext}}\right)+\left(\frac{q}{m} F_{\beta \sigma}^{\mathrm{ext}}\right) \frac{q}{m} F_{\delta}^{\mathrm{ext} \sigma} u^{\delta}\right]
$$

is known as the "reduced order" Abraham-Lorentz equation. This equation is free from the "runaway" solutions that plague eq.(9), and has a standard, second-order initial value formulation. Thus, eq. (12) appears to be satisfactory on both mathematical and physical grounds. However, the reduction of order procedure has the appearance of being rather ad $h o c$, and if eq. (9) is, in some sense, more fundamentally correct, it is difficult to justify replacing this equation with eq. (12) other than on purely pragmatic grounds.

Our analysis sheds considerable light on this issue. We will prove in section IV that the first order deviation from Lorentz force motion is given by ${ }^{5}$

$$
\delta\left[\hat{m} a_{\alpha}\right]=\delta\left[q F_{\alpha \beta}^{\mathrm{ext}} u^{\beta}\right]+\left(g_{\alpha}{ }^{\beta}+u_{\alpha} u^{\beta}\right)\left\{\frac{2}{3} q^{2} \frac{D}{d \tau} a_{\beta}-\frac{1}{2} Q^{\gamma \delta} \nabla_{\beta} F_{\gamma \delta}^{\mathrm{ext}}+\frac{D}{d \tau}\left(a^{\gamma} S_{\gamma \beta}-2 u^{\delta} Q_{[\beta}^{\gamma} F_{\delta] \gamma}^{\mathrm{ext}}\right)\right\} .
$$

In this equation $\delta[\ldots]$ refers to the first perturbative correction of ..., whereas all other quantities are lowest non-vanishing order. The parameters $q, \hat{m}, Q^{\alpha \beta}$, and $S^{\alpha \beta}$ are, respectively, the charge, mass, ${ }^{6}$ electromagnetic dipole moment, and spin of the body. Here the electromagnetic dipole $Q^{\alpha \beta}$ can be decomposed in terms of the electric dipole moment $p^{\alpha}$ and magnetic dipole moment $\mu^{\alpha}$ by

$$
Q^{\alpha \beta}=2 u^{[\alpha} p^{\beta]}-\epsilon^{\alpha \beta \gamma \delta} u_{\gamma} \mu_{\delta}
$$

with $u^{\alpha} p_{\alpha}=u^{\alpha} \mu_{\alpha}=0$, and the spin tensor $S^{\alpha \beta}$ can be expressed in terms of the spin vector $S^{\alpha}$ by

$$
S^{\alpha \beta}=\epsilon^{\alpha \beta \gamma \delta} u_{\gamma} S_{\delta}
$$

\footnotetext{
${ }^{5}$ We also obtain equations for the time evolution of $S_{a b}$ and $\delta m$ (see eqs. (135) and (136) below). However, the electromagnetic dipole $Q^{a b}$ does not have any associated conservation law and can be "changed at will by the body itself", so there exists no universal evolution equation for this quantity. Similar remarks should hold for all higher moments (of mass and of charge), although these moments would show up only at higher orders in our perturbation expansion.

${ }^{6}$ The perturbed mass, $\delta \hat{m}$, includes a contribution from the interaction energy of the electric dipole with the external field (see eq.(131) below).
} 
The first term on the right side of equation (13) is the first order correction to the ordinary Lorentz force law. The first term in braces corresponds to the ALD self-force. The second term in braces is a relativistic form of the usual dipole forces familiar from electrostatics and magnetostatics. The remaining terms are additional dipole and spin forces relevant in nonstationary situations. A non-relativistic version of these force terms is given in eq.(143) below.

In the case of a body with negligible electric and magnetic dipole moments and negligible spin, only the first two terms of equation (13) remain. In this case, we see that the form of the ALD equation (9) is recovered as the leading order perturbative correction to Lorentz force motion for a sufficiently small charged body. However, since the acceleration $a^{\mu}$ present in the ALD term refers to the "background motion" (i.e., a solution to the Lorentz force equations of motion in the background electromagnetic field), no runaway behavior occurs in the context of perturbation theory.

Although (13) has been rigorously derived as the leading order perturbative correction to Lorentz force motion, small deviations from Lorentz force motion can accumulate over time. Eventually, the deviation from the zeroth order motion should become large, so one would not expect that solutions to (13) would provide an accurate description of motion at late times. However, as argued in [9], if the deviations from Lorentz force motion are locally small, it may be possible to find a "self-consistent perturbative equation" based upon eqs.(5) and (13) that provides a good, global-in-time description of the motion. We shall argue in section $\nabla$ that in the case of a body with negligible electric and magnetic dipole moments and negligible spin, the reduced order Abraham-Lorentz equation (12) provides such a desired self-consistent perturbative equation. This provides a solid justification for using eq.(12) to describe the motion of sufficiently small charged bodies (with negligible dipole moments and spin). There is no corresponding justification for using the ALD equation (99) to describe the motion of charged bodies.

Our notation and conventions are as follows. Greek indices $\mu, \nu, .$. will refer to spacetime coordinate components, whereas mid-alphabet Latin indices $i, j, \ldots$ will refer to spatial coordinates only. A "0" will denote the time component. Early-alphabet Latin indices $a, b, \ldots$ will refer to abstract spacetime indices [12]. (Typically, $a, b, \ldots$ will be used in diffeomorphism covariant expressions, whereas $\mu, \nu, \ldots$ will be used for expressions that take a special form in the coordinates being used.) Our (flat) metric $g_{a b}$ has signature $(-1,1,1,1)$. We 
normalize the Levi-Civita symbol $\epsilon_{a b c d}$ so that $\epsilon_{0123}=+1$.

\section{ASSUMPTIONS}

We consider a one-parameter family of charged bodies described by a charge-current vector $J^{\mu}(\lambda)$ and a matter stress-energy tensor $T_{\mu \nu}^{M}(\lambda)$. The electromagnetic field $F_{\mu \nu}(\lambda)$ is assumed to satisfy Maxwell's equations (11) and (2), the charge-current is assumed to be conserved, eq.(3), and the total stress energy tensor is assumed to be conserved, eq.(7). Since we seek relations that hold universally for all bodies, we specify no additional "constitutive" relations, and impose only eqs.(11)-(3) and (77) in our analysis.

As discussed in the previous section, we wish to consider a limit where the body shrinks down to zero size, but in such a way that the mathematical (and physical) inconsistencies associated with point particles are avoided. Following the basic ideas of [9], this can be done by considering a limit where not only the size of the body goes to zero, but its charge and mass also go to zero in such a way that, asymptotically, the body continues to "look the same" except for its overall scale. In [9], this idea was implemented in a relatively indirect way by postulating the existence of a "scaled limit" of the exterior field of the body together with the existence of an "ordinary limit" and a "uniformity condition" on these limits. In the context of general relativity, it was necessary in [9] to formulate the conditions on the one-parameter family in terms of the exterior field because we wished to consider strong field objects (such as black holes), which do not admit a simple description in terms of a stress-energy source. However, in the present context of classical electrodynamics in Minkowski spacetime, all of the relevant information about the body and the electromagnetic field is contained in the specification of the charge current density $J^{\mu}$, the matter stressenergy tensor $T_{\mu \nu}^{M}$, and "external" electromagnetic field, i.e., the homogeneous solution of Maxwell's equations that describes the difference between the actual electromagnetic field and the retarded solution associated with $J^{\mu}$. Consequently, we will formulate our conditions in terms of these quantities.

We wish to consider a one-parameter family $\left\{F_{\mu \nu}(\lambda), J^{\mu}(\lambda), T_{\mu \nu}^{M}(\lambda)\right\}$ of solutions to eqs.(1)-(3) and (77) having the property that the worldtube, $\mathcal{W}(\lambda)$, containing the supports of $J^{\mu}(\lambda)$ and $T_{\mu \nu}^{M}(\lambda)$ shrinks down to a timelike worldline $\gamma$ as $\lambda \rightarrow 0$. Furthermore, we wish to impose the condition that this "shrinking down" asymptotically corresponds merely to a 
"change of scale". To get a feeling for what this entails, fix a time, $t_{0}$, on $\gamma$, and consider the charge density, $\rho\left(\lambda, x^{i}\right)$, on the hyperplane orthogonal to $\gamma$ at $t_{0}$ given by

$$
\rho\left(\lambda, x^{i}\right)=\lambda^{-2} \tilde{\rho}\left(\frac{x^{i}-z^{i}}{\lambda}\right)
$$

where $\tilde{\rho}$ is a smooth function on $\mathbf{R}^{3}$ that vanishes for $r>R$ for some $R>0$. Then $\rho\left(\lambda, x^{i}\right)$ vanishes for $r>\lambda R$, i.e., the support of $\rho$ shrinks to $z^{i}$ as $\lambda \rightarrow 0$. It also is clear that for the one-parameter family, eq.(16), the charge density "retains its shape" exactly as it shrinks to the origin. Finally, the overall scaling of $\lambda^{-2}$ in eq.(16) ensures that the total charge also goes to zero proportionally to $\lambda$. Thus, eq.(16) describes the kind of "scaling to zero size" that we seek.

However, since a body undergoing non-uniform motion cannot remain "rigid", we must allow for time dependence in the manner in which the charge-current and body stress-energy scale to zero size, particularly in view of the fact that the body may find itself in a different external field at different times. In addition, the exact scaling represented by eq.(16) is too strong an assumption; in particular, it could hold, at most, for a particular choice of time slicing and a particular choice of spatial coordinates on these slices. Furthermore, although we could always impose eq.(16) exactly as an initial condition at one time, there is no reason to believe that dynamical evolution would preserve this exact scaling at later times. Instead, it would be much more reasonable to require that the type of scaling represented by eq.(16) hold only asymptotically as $\lambda \rightarrow 0$.

In order to formulate our conditions on $J^{\mu}(\lambda)$ and $T_{\mu \nu}^{M}(\lambda)$, it is useful to adopt a specific choice of coordinate system, namely Fermi normal coordinates (see, e.g., [13]). These coordinates $\left(T, X^{i}\right)$ are defined with reference to a timelike worldline, $\gamma$. The surfaces of constant $T$ are orthogonal to $\gamma$, and the $X^{i}$ measure spatial distance within those surfaces. With this coordinate choice we may state our conditions on $J^{\mu}(\lambda)$ and $T_{\mu \nu}^{M}(\lambda)$ as follows:

- (i) We require that there be a smooth, timelike worldline $\gamma$ such that in Fermi normal coordinates, $\left(T, X^{i}\right)$, based on $\gamma$, we have $J^{\mu}\left(\lambda, T, X^{i}\right)=\lambda^{-2} \tilde{J}^{\mu}\left(\lambda, T, X^{i} / \lambda\right)$ and $T_{\mu \nu}^{M}\left(\lambda, T, X^{i}\right)=\lambda^{-2} \tilde{T}_{\mu \nu}^{M}\left(\lambda, T, X^{i} / \lambda\right)$, where $\tilde{T}^{M}$ and $\tilde{J}$ are smooth functions of their arguments. Furthermore, the supports of $\tilde{T}^{M}$ and $\tilde{J}$ in the last variables-i.e., the slots into which $X^{i} / \lambda$ have been inserted-are contained within a radius $R$, so that the $J^{\mu}(\lambda)$ and $T_{\mu \nu}^{M}(\lambda)$ are nonvanishing only within a worldtube, $\mathcal{W}(\lambda)$, of radius $\lambda R$ 
about $\gamma$.

Note that the smooth dependence on both $T$ and $\lambda$ permitted for $\tilde{J}^{\mu}$ and $\tilde{T}_{\mu \nu}^{B}$ in this condition addresses the concerns of the previous paragraph.

We now present these assumptions in general coordinates. Let $\left(t, x^{i}\right)$ denote any coordinates that are smoothly related to Fermi normal coordinates in the neighborhood of $\gamma$ in which the Fermi normal coordinates are defined and are such that $\nabla_{\mu} t$ is timelike. In these new coordinates, the worldline $\gamma$ will be given by an equation of the form $x^{i}=z^{i}(t)$. Since the Fermi coordinate $X^{i}$ is a smooth function that vanishes on $\gamma$, we must have

$$
X^{i}=\sum_{j}\left[x^{j}-z^{j}(t)\right] h_{i j}\left(t, x^{k}\right)
$$

where $h_{i j}$ is smooth. Writing $T=T\left(t, x^{k}\right)$, writing

$$
x^{k}=\lambda \frac{x^{k}-z^{k}(t)}{\lambda}+z^{k}(t)
$$

and substituting into the formula for the charge current density in the new coordinates, we find that the components of the charge current density in the new coordinates take the form

$$
J^{\mu}\left(\lambda, t, x^{i}\right)=\lambda^{-2} \tilde{J}^{\prime \mu}\left(\lambda, t,\left[x^{i}-z^{i}(t)\right] / \lambda\right)
$$

where $\tilde{J}^{\prime \mu}$ is a smooth function of its arguments. Furthermore, since $\nabla_{\mu} t$ is timelike, each surface of constant $t$ must intersect $\mathcal{W}(\lambda)$ in a compact set, which implies that $\tilde{J}^{\prime \prime}$ is of compact support in the last variables. Note that, in particular, the form eq.(19) holds in global inertial coordinates. By reversing the argument, it is easily seen that assumption (i) on $J^{\mu}(\lambda)$ as stated in Fermi normal coordinates is equivalent to eq.(19) holding in some coordinates with $\nabla_{\mu} t$ timelike, together with the requirement that the support of $J^{\mu}(\lambda)$ be contained in a worldtube of proper distance $\lambda R$ from $\gamma$. Similar results hold, of course, for $T_{\mu \nu}^{M}\left(\lambda, t, x^{i}\right)$.

Our assumption about the electromagnetic field $F_{\mu \nu}(\lambda)$ is as follows:

- (ii) We have $F_{\mu \nu}=F_{\mu \nu}^{\text {ext }}+F_{\mu \nu}^{\text {self }}$, where $F_{\mu \nu}^{\text {self }}$ is the retarded ${ }^{7}$ solution of Maxwell's

\footnotetext{
${ }^{7}$ It may appear that we are introducing a time asymmetry in our assumptions by choosing $F_{\mu \nu}^{\text {self }}$ to be the
} 
equations with source $J^{\mu}(\lambda)$ and $F_{\mu \nu}^{\text {ext }}$ is a homogeneous solution of Maxwell's equation that is jointly smooth function of $\lambda$ and the spacetime point.

Assumptions (i)-(ii) about our one-parameter family together with eqs.(11)-(3) and (7) constitute the entirety of what we shall assume in this paper.

In Appendix $\mathrm{A}$, we show that in arbitrary smooth coordinates $\left(t, x^{i}\right)$, the retarded solution, $F_{\mu \nu}^{\text {self }}(\lambda)$, with source of the form eq.(19) takes the form

$$
F_{\mu \nu}^{\text {self }}\left(\lambda, t, x^{i}\right)=\lambda^{-1} \tilde{F}_{\mu \nu}\left(\lambda, t,\left[x^{i}-z^{i}(t)\right] / \lambda\right)
$$

where $\tilde{F}$ is a smooth function of its arguments.

Finally, we relate the assumptions about our one-parameter family made here to the assumptions made in [9]. As previously mentioned, the assumptions of [9] were formulated entirely in terms of the behavior of the "exterior field" of the body, i.e., the behavior of the spacetime metric outside of the body. Assumption (i) of [9] ("existence of the ordinary limit") corresponds here to the requirement that $F_{\mu \nu}$ be jointly smooth in $\left(\lambda, t, x^{i}\right)$ except on $\gamma$ and that $F_{\mu \nu}(\lambda=0)$ be smoothly extendible to $\gamma$. That this condition holds follows immediately from our assumption (ii) together with the fact that $F_{\mu \nu}^{\text {self }}$ goes to zero at fixed $\left(t, x^{i}\right)$ with $x^{i} \neq z^{i}(t)$ as $\lambda \rightarrow 0$. The analog of assumption (ii) of [9] ("existence of the scaled limit") follows immediately from assumption (ii) and eq.(20). We will consider this scaled limit in detail in section \V below. Finally, the analog of the "uniformity condition" (iii) of [9] is as follows: Define $\alpha=r=\sqrt{\sum\left[x^{i}-z^{i}(t)\right]^{2}}$ and define $\beta=\lambda / r$. Write $F_{\mu \nu}$ as a function of $(\alpha, \beta, t, \theta, \phi)$. The desired condition is that at fixed $(t, \theta, \phi), \lambda F_{\mu \nu}$ is jointly smooth in $(\alpha, \beta)$ at $(0,0)$. Since $\lambda F_{\mu \nu}^{\text {ext }}$ is easily seen to satisfy this condition, the analog of the uniformity condition of [9] will hold here provided that $\tilde{F}_{\mu \nu}$ is smooth in $(\alpha, \beta)$ at $(0,0)$ at fixed $(t, \theta, \phi)$. In appendix $\mathrm{A}$, we show that not only is $\tilde{F}_{\mu \nu}$ smooth in $(\alpha, \beta)$, but is of the form of $\beta^{2}$ times a smooth function of $(\alpha, \beta)$.

Thus, the assumptions we have made above imply analogs of the assumptions made in [9]. Since our assumptions here are quite simple and straightforward as compared with the assumptions made in [9], this may be viewed as providing justification for the assumptions

retarded solution. However, choosing $F_{\mu \nu}^{\text {self }}$ to be the advanced solution results in an equivalent set of assumptions, since the retarded minus advanced solution is a homogeneous solution that can be shown to be smooth in $\left(\lambda, t, x^{i}\right)$. 
made in [9]. Since the assumptions (i)-(iii) of [9] are essentially what is needed to justify the use of "matched asymptotic expansions" [13, 14], we have thus effectively also provided justification for the use matched asymptotic expansions.

\section{THE FAR-ZONE LIMIT AND LORENTZ FORCE MOTION}

As in [9], as $\lambda \rightarrow 0$ it will be useful to consider both a "far-zone" limit (wherein $x^{\mu}$ is held fixed) and a "near zone" limit (wherein $\bar{x}^{\mu} \equiv\left(\left[t-t_{0}\right] / \lambda,\left[x^{i}-z^{i}\left(t_{0}\right)\right] / \lambda\right)$ is held fixed). Roughly speaking, the "far zone" limit corresponds to how the body and the electromagnetic field appear to an observer at a fixed radius from $\gamma$ as the body shrinks down to $\gamma$. The near zone limit describes the appearance to an observer who "follows the body in" toward $\gamma$ at time $t_{0}$ - and correspondingly rescales units - as the body shrinks. We use the terminology "far zone" and "near zone" because of the close correspondence to the way these terms are used in matched asymptotic expansion analyses. However, we will not need to do any "matching" in our analysis, and any of our calculations can be done in either the "far" or "near" zone pictures. In this section, we treat the far-zone limit. We will show that at first order in $\lambda$, the far zone description of the body is that of a point particle, and that the worldline $\gamma$ satisfies the Lorentz force equation of motion (5). Interestingly, we will also see that the electromagnetic self-energy of the body makes a non-zero, finite contribution to the particle's mass.

It is clear from assumption (i) of the previous section that $J^{\mu}(\lambda)$ and $T_{\mu \nu}^{M}(\lambda)$ as well as all of their $\lambda$-derivatives go to zero pointwise as $\lambda \rightarrow 0$ and any fixed $x^{\mu} \notin \gamma$. On the other hand, $J^{\mu}(\lambda)$ and $T_{\mu \nu}^{M}(\lambda)$ and their $\lambda$-derivatives do not approach any limit at all for $x^{\mu} \in \gamma$. Nevertheless, $J^{\mu}(\lambda), T_{\mu \nu}^{M}(\lambda)$ and their $\lambda$-derivatives have well defined limits as distributions as $\lambda \rightarrow 0$. To see this, we recall that associated to any locally $L^{1}$ function $H\left(t, x^{i}\right)$ on spacetime is the distribution, $D_{H}$, defined by the following action on a smooth test function $f\left(t, x^{i}\right)$ of compact support,

$$
D_{H}[f]=\langle H, f\rangle=\int H\left(t, x^{i}\right) f\left(t, x^{i}\right) \sqrt{-g} d^{4} x
$$


Thus, viewed as a distribution, the charge-current $J^{\mu}(\lambda)$ is given by

$$
\begin{aligned}
D_{J}(\lambda)[f] & =\int J^{\mu}\left(\lambda, t, x^{i}\right) f_{\mu}\left(t, x^{i}\right) \sqrt{-g} d t d^{3} x \\
& =\int \lambda^{-2} \tilde{J}^{\mu}\left(\lambda, t,\left[x^{i}-z^{i}(t)\right] / \lambda\right) f_{\mu}\left(t, x^{i}\right) \sqrt{-g} d t d^{3} x \\
& =\lambda \int \tilde{J}^{\mu}\left(\lambda, t, \bar{x}^{i}\right) f_{\mu}\left(t, z^{i}(t)+\lambda \bar{x}^{i}\right) \sqrt{-g} d t d^{3} \bar{x} \\
& =\lambda \int d t \frac{d \tau}{d t} f_{\mu}\left(t, z^{i}(t)\right) \int d^{3} \bar{x} \sqrt{g_{3}} \tilde{J}^{\mu}\left(0, t, \bar{x}^{i}\right)+O\left(\lambda^{2}\right) .
\end{aligned}
$$

(Here we work in arbitrary smooth coordinates $\left(t, x^{i}\right)$ and have used eq.(19) in the second line; we also have dropped the prime on $\tilde{J}^{\mu}$.) Thus, we see that, viewing $J^{\mu}(\lambda)$ as a oneparameter family of distributions, we have

$$
\begin{aligned}
J^{(0) \mu} & \equiv \lim _{\lambda \rightarrow 0} J^{\mu}(\lambda)=0 \\
J^{(1) \mu} & \equiv \lim _{\lambda \rightarrow 0} \frac{\partial}{\partial \lambda} J^{\mu}(\lambda)=\mathcal{J}^{\mu}(t) \frac{\delta\left(x^{i}-z^{i}(t)\right)}{\sqrt{-g}} \frac{d \tau}{d t},
\end{aligned}
$$

with

$$
\mathcal{J}^{\mu}(t) \equiv \int \tilde{J}^{\mu}\left(\lambda=0, t, \bar{x}^{i}\right) \sqrt{g_{3}} d^{3} \bar{x}
$$

Higher-order derivatives of $J^{\mu}$ with respect to $\lambda$ will similarly have the form of distributions with support on $\gamma$. For example, $J^{(2) \mu}$ will contain a delta function, which will contribute a correction to the charge of the body, along with a derivative of delta function, which will provide the leading order contribution to the electromagnetic dipole moment. In general, the $n$th order charge-current $J^{(n) \mu}$ will have the form of the sum of derivatives of the delta function up to order $n$, where the coefficient of the $m$ th order delta function is the $(n-m)$ th correction to the $(n-1)$ st moment.

We can obtain considerably more information about the form of $J^{(1) \mu}$, eq.(24), by making use of conservation of $J^{\mu}$, eq.(3). For any test function $f$, we have

$$
\int \nabla_{\nu} J^{\nu}(\lambda) f \sqrt{-g} d^{4} x=0
$$

for all $\lambda>0$. Integrating by parts, differentiating with respect to $\lambda$, and taking the limit as 
$\lambda \rightarrow 0$, we obtain

$$
\int \mathcal{J}^{\mu}(t)\left[\nabla_{\mu} f\right]\left(t, z^{i}(t)\right) d \tau=0
$$

for all test-functions $f$. Now, for any $i=1,2,3$, choose $f$ to be a test-function of the form $f=X^{i} c(t) h\left(X^{j}\right)$, where $X^{i}$ is a Fermi normal spatial coordinate and $c$ and $h$ are of compact support with $h\left(X^{j}\right)=1$ in a neighborhood of the origin. Equation (27) then yields

$$
\int \mathcal{J}^{\mu}(t)\left[\nabla_{\mu} X^{i}\right] c(t) d \tau=0
$$

which implies that the projection of $\mathcal{J}^{\mu}(t)$ orthogonal to $\gamma$ vanishes for all $t$, i.e., $\mathcal{J}^{\mu}$ is of the form $\mathcal{J}(t) u^{\mu}$. Using this result in equation (27) and integrating by parts, we find that $\dot{\mathcal{J}}(t)=0$. We thus obtain

$$
J^{(1) \mu}=q u^{\mu} \frac{\delta\left(x^{i}-z^{i}(t)\right)}{\sqrt{-g}} \frac{d \tau}{d t},
$$

where the (lowest-order) total charge $q$ is given by

$$
q \equiv \int \tilde{J}^{0}\left(\lambda=0, t, \bar{x}^{i}\right) \sqrt{g_{3}} d^{3} \bar{x}
$$

and is independent of time $t$. Thus, to first order in $\lambda$, the charge current of the body is precisely the standard form of a structureless point charge moving on the worldline $\gamma$.

The electromagnetic field $F_{\mu \nu}$ is the sum of $F_{\mu \nu}^{\text {ext }}$ and $F_{\mu \nu}^{\text {self }}$. The external field $F_{\mu \nu}^{\text {ext }}$ is smooth in $\lambda$ and satisfies the homogeneous Maxwell's equations at all $\lambda$. Therefore, $F_{\mu \nu}^{\text {ext }}(\lambda)$ has a straightforward perturbation expansion in $\lambda$, and all $\lambda$-derivatives of $F_{\mu \nu}^{\text {ext }}$ satisfy the homogeneous Maxwell's equations at each order in perturbation theory.

From eq. (A22) of appendix $\mathrm{A}$, we see that the self-field is of the form

$$
F_{\mu \nu}^{\text {self }}=\frac{\lambda}{r^{2}} \mathcal{F}_{\mu \nu}(t, r, \lambda / r, \theta, \phi),
$$

where, for any fixed $(t, \theta, \phi), \mathcal{F}_{\mu \nu}$ is smooth near 0 in its second and third arguments. Using 
this property, we may Taylor expand $F_{\mu \nu}^{\text {self }}$ to any finite orders $N, M$,

$$
\begin{aligned}
F_{\mu \nu}^{\mathrm{self}}(t, r, \theta, \phi) & =\frac{\lambda}{r^{2}} \sum_{n=0}^{N} \sum_{m=0}^{M} r^{n}\left(\frac{\lambda}{r}\right)^{m}\left(\mathcal{F}_{\mu \nu}\right)_{n m}(t, \theta, \phi)+O\left(r^{N+1}\right)+O\left((\lambda / r)^{M+1}\right) \\
& =\sum_{n=0}^{N} \sum_{m=0}^{M} \lambda^{m+1} r^{n-m-2}\left(\mathcal{F}_{\mu \nu}\right)_{n m}(t, \theta, \phi)+O\left(r^{N+1}\right)+O\left((\lambda / r)^{M+1}\right)
\end{aligned}
$$

This gives the general form of the far-zone expansion of $F_{\mu \nu}^{\text {self }}$ near $r=0$.

We can obtain explicit forms for the terms arising in the far-zone expansion of $F_{\mu \nu}^{\text {self }}$ by using the fact that for all $\lambda, F_{\mu \nu}^{\text {self }}(\lambda)$ is the retarded solution associated with the chargecurrent $J^{\mu}(\lambda)$, so the $n$-order perturbative self-field $F_{\mu \nu}^{\text {self, }(n)}$ is the retarded solution associated with the distributional source $J^{(n) \mu}$. In particular, the zeroth order self-field vanishes

$$
F_{\mu \nu}^{\text {self, }(0)}=0
$$

and the first order self-field $F_{\mu \nu}^{\text {self,(1) }}$ is just the standard Lienard-Wiechert solution associated with the point charge source, eq.(24). In Fermi normal coordinates about $\gamma, F_{\mu \nu}^{\text {self, }(1)}$ takes the explicit form

$$
\begin{aligned}
F_{i 0}^{\text {self, }(1)}=q & \left\{\frac{n_{i}}{r^{2}}+\frac{1}{r}\left(-\frac{1}{2}\left(a_{j} n^{j}\right) n_{i}-\frac{1}{2} a_{i}\right)+\right. \\
& \left.+\frac{3}{8}\left(a_{j} n^{j}\right)^{2} n_{i}+\frac{3}{4}\left(a_{j} n^{j}\right) a_{i}+\frac{1}{8} a_{j} a^{j} n_{i}+\frac{1}{2} \dot{a}_{0} n_{i}+\frac{2}{3} \dot{a}_{i}+O(r)\right\} \\
F_{i j}^{\text {self },(1)}=- & q \frac{1}{2} \dot{a}_{[i} n_{j]}+O(r),
\end{aligned}
$$

where $n^{i}=x^{i} / r$.

We now turn our attention to the stress-energy tensor. By assumption (i), the matter stress-energy $T_{\mu \nu}^{M}(\lambda)$ has the same type of behavior as $J^{\mu}(\lambda)$ as $\lambda \rightarrow 0$, and thus it has an analogous multipole series. In particular, as a distribution, $T_{\mu \nu}^{M}(\lambda) \rightarrow 0$ as $\lambda \rightarrow 0$, whereas to first order in $\lambda$, the matter stress-energy tensor takes the form

$$
T_{\mu \nu}^{M,(1)} \equiv \lim _{\lambda \rightarrow 0} \frac{\partial}{\partial \lambda} T_{\mu \nu}^{M}(\lambda)=\mathcal{T}_{\mu \nu}^{M}(t) \frac{\delta\left(x^{i}-z^{i}(t)\right)}{\sqrt{-g}} \frac{d \tau}{d t}
$$

The electromagnetic stress energy tensor is given by eq.(8), with $F_{\mu \nu}=F_{\mu \nu}^{\text {ext }}+F_{\mu \nu}^{\text {self }}$. Thus, 
the total electromagnetic stress-energy naturally breaks up into a sum of three terms,

$$
T_{\mu \nu}^{E M}=T_{\mu \nu}^{\mathrm{ext}}+T_{\mu \nu}^{\mathrm{cross}}+T_{\mu \nu}^{\mathrm{self}}
$$

where the notation should be self-explanatory. The stress-energy tensor $T_{\mu \nu}^{\text {ext }}$ of the external field $F_{\mu \nu}^{\text {ext }}$ behaves smoothly in $\left(\lambda, x^{\mu}\right)$ and thus has a straightforward perturbative expansion in $\lambda$. Since $F_{\mu \nu}^{\text {ext }}$ is a solution to the homogeneous Maxwell's equations, we also have $\nabla^{\mu} T_{\mu \nu}^{\operatorname{ext}}(\lambda)=0$ for all $\lambda$, so conservation of $T_{\mu \nu}^{\text {ext, }(n)}$ holds at each order in perturbation theory.

The behavior of the stress-energy tensor $T_{\mu \nu}^{\text {self }}$ of the self-field $F_{\mu \nu}^{\text {self }}$ as $\lambda \rightarrow 0$ is more delicate. Since $F_{\mu \nu}^{\text {self, }(0)}=0$, one might expect that the lowest order contribution to $T_{\mu \nu}^{\text {self }}$ would be obtained by substituting $\lambda F_{\mu \nu}^{\text {self,(1) }}$, eq.(34), into eq.(86). This would give an expression of the form $\lambda^{2}$ times the usual stress-energy tensor of a point charge. This would suggest that the first order in $\lambda$ contribution to $T_{\mu \nu}^{\text {self }}$ vanishes, and the second order contribution is too singular on $\gamma$ to define a distribution. If this were the case, then our analysis would be plagued by the same type of infinite self-energy problems that occur in usual treatments of point particles. Fortunately, as we now shall show, a more careful treatment shows that to first order in $\lambda, T_{\mu \nu}^{\text {self }}$ has a non-zero, well defined distributional limit.

As previously mentioned in the Introduction, in Appendix $\mathrm{A}$ we prove that $F_{\mu \nu}^{\text {self }}$ is of the form (20), where $\tilde{F}_{\mu \nu}$ is a smooth function of $\left(\lambda, t,\left[x^{i}-z^{i}(t)\right] / \lambda\right)$. We also prove in Appendix A that if we define $\alpha=r=\sqrt{\sum\left[x^{i}-z^{i}(t)\right]^{2}}$, define $\beta=\lambda / r$, and write $\tilde{F}_{\mu \nu}$ as a function of $(\alpha, \beta, t, \theta, \phi)$, then, at any fixed $(t, \theta, \phi), \tilde{F}_{\mu \nu}$ is of the form of $\beta^{2}$ times a smooth function of $(\alpha, \beta)$ at $(0,0)$. Consequently, $T_{\mu \nu}^{\text {self }}$ is of the form

$$
T_{\mu \nu}^{\mathrm{self}}\left(\lambda, t, x^{i}\right)=\lambda^{-2} \tilde{T}_{\mu \nu}^{\mathrm{self}}\left(\lambda, t,\left[x^{i}-z^{i}(t)\right] / \lambda\right)
$$

where $\tilde{T}_{\mu \nu}^{\text {self }}$ is given in terms of $\tilde{F}_{\mu \nu}$ by eq.(8) and is a smooth function of its arguments. Thus, $\tilde{T}_{\mu \nu}^{\text {self }}(\lambda)$ is of a form similar to that of $J^{\mu}(\lambda)$ and $T_{\mu \nu}^{M}(\lambda)$ in our condition (i) of the previous section, except that it is not of compact support in $x^{i}$. Nevertheless, from the properties of $\tilde{F}_{\mu \nu}$, we know that at any fixed $(t, \theta, \phi), \tilde{T}_{\mu \nu}^{\text {self }}$ is of the form of $\beta^{4}$ times a smooth function of $(\alpha, \beta)$. If we view $T_{\mu \nu}^{\text {self }}(\lambda)$ for $\lambda>0$ as a distribution, the same manipulations as led to 
eq.(22) now yield

$$
D_{T^{\text {self }}}[f]=\lambda \int \tilde{T}_{\mu \nu}^{\text {self }}\left(\lambda, t, \bar{x}^{i}\right) f^{\mu \nu}\left(t, z^{i}(t)+\lambda \bar{x}^{i}\right) \sqrt{-g} d t d^{3} \bar{x}
$$

If $\tilde{T}_{\mu \nu}^{\text {self }}$ were of compact support in $\bar{x}^{i}$, we could straightforwardly take the limit as $\lambda \rightarrow 0$ inside the integral, as we did in eq.(22). Nevertheless, using the facts that (1) the integrand is smooth in $\bar{x}^{i},(2)$ the test tensor field $f^{\mu \nu}$ is of compact support in $x^{i}$ (and, thus, is of compact support in $\alpha$ ) and (3) for $\alpha$ in a compact set, $\tilde{T}_{\mu \nu}^{\text {self }}$ is bounded by $C \beta^{4}$ for some constant $C$, it is not difficult to show that for $\lambda \leq \lambda_{0}$

$$
\left|\tilde{T}_{\mu \nu}^{\text {self }}\left(\lambda, t, \bar{x}^{i}\right)\right|\left|f^{\mu \nu}\left(t, z^{i}(t)+\lambda \bar{x}^{i}\right)\right| \leq \frac{K}{\bar{r}^{4}+1}
$$

where $K$ is a constant (i.e., independent of $\lambda$ and $x^{i}$ ). Since $1 /\left[\bar{r}^{4}+1\right]$ is integrable with respect to $\sqrt{-g} d^{3} \bar{x}$, the dominated convergence theorem then allows us to take the limit as $\lambda \rightarrow 0$ inside the integral in this case as well, and we obtain

$$
D_{T^{\text {self }}}[f]=\lambda \int d t \frac{d \tau}{d t} f^{\mu \nu}\left(t, z^{i}(t)\right) \int d^{3} \bar{x} \sqrt{g_{3}} \tilde{T}_{\mu \nu}^{\text {self }}\left(0, t, \bar{x}^{i}\right)+O(\lambda)
$$

i.e., as distributions we have

$$
\begin{aligned}
& T_{\mu \nu}^{\text {self,}(0)} \equiv \lim _{\lambda \rightarrow 0} T_{\mu \nu}^{\text {self }}(\lambda)=0 \\
& T_{\mu \nu}^{\text {self, }(1)} \equiv \lim _{\lambda \rightarrow 0} \frac{\partial}{\partial \lambda} T_{\mu \nu}^{\text {self }}(\lambda)=\mathcal{T}_{\mu \nu}^{\text {self }}(t) \frac{\delta\left(x^{i}-z^{i}(t)\right)}{\sqrt{-g}} \frac{d \tau}{d t}
\end{aligned}
$$

with

$$
\mathcal{T}_{\mu \nu}^{\text {self }}(t) \equiv \int \tilde{T}_{\mu \nu}\left(0, t, \bar{x}^{i}\right) \sqrt{g_{3}} d^{3} \bar{x}
$$

Thus, despite the fact that the electromagnetic self-field is not confined to a world-tube at any $\lambda>0$, to first order in $\lambda$, the electromagnetic self-stress-energy takes the form of a $\delta$-function on $\gamma$. However, it should be noted that, in contrast to the situation for $J^{\mu}$ and $T_{\mu \nu}^{M}$, at higher orders in $\lambda$ the support of $T_{\mu \nu}^{\text {self }}$ cannot be confined to $\gamma$. Indeed, although we have shown above that $D_{T^{\text {self }}}[f]$, eq.(38), is a $C^{1}$ function of $\lambda$ at $\lambda=0$, it does not appear 
that it is $\operatorname{smooth}^{8}$ in $\lambda$ at $\lambda=0$.

There is nothing further that we can say beyond eqs.(35) and (42) about the individual forms of the material stress-energy and electromagnetic self-stress-energy to first order in $\lambda$. However, defining the "matter plus electromagnetic self-field" stress-energy $T_{\mu \nu}$,

$$
T_{\mu \nu} \equiv T_{\mu \nu}^{M}+T_{\mu \nu}^{\mathrm{self}}
$$

we see from conservation of total stress-energy, (7), that for all $\lambda$ we have

$$
\begin{aligned}
\nabla^{\mu} T_{\mu \nu} & =-\nabla^{\mu} T_{\mu \nu}^{\mathrm{ext}}-\nabla^{\mu} T_{\mu \nu}^{\mathrm{cross}} \\
& =F_{\mu \nu}^{\mathrm{ext}} J^{\nu}
\end{aligned}
$$

where we emphasize that $T_{\mu \nu}$, with no superscript, includes the matter and "electromagnetic self-field" contributions, as in equation (44). The same type of calculation that led from eq.(26) to eq.(29) then gives to first order in $\lambda$ that

$$
T_{\mu \nu}^{(1)}(t)=m u_{\mu} u_{\nu} \frac{\delta\left(x^{i}-z^{i}(t)\right)}{\sqrt{-g}} \frac{d \tau}{d t}
$$

where $u^{\mu}$ is the unit tangent to $\gamma$, and $m$ is a constant (i.e.,independent of $t$ ). In coordinates $\left(t, x^{i}\right)$ such that the constant-t hyperplanes are orthogonal to $u^{\mu}, m$ is given by

$$
m=\int\left(\tilde{T}_{00}^{M}\left(\lambda=0, t, \bar{x}^{i}\right)+\tilde{T}_{00}^{\text {self }}\left(\lambda=0, t, \bar{x}^{i}\right)\right) \sqrt{g_{3}} d^{3} \bar{x}
$$

Furthermore, $u^{\mu}$ must satisfy

$$
m u^{\nu} \nabla_{\nu} u_{\mu}=q u^{\nu} F_{\mu \nu}^{\mathrm{ext}}\left(\lambda=0, t, z^{i}(t)\right)
$$

We may summarize what we have just shown as follows: Consider any one-parameter family of bodies satisfying the assumptions of the previous section. Then, to first order in $\lambda$, the description of the body is precisely that of a classical point charge/mass moving on

\footnotetext{
${ }^{8}$ A failure in obtaining a distributional series for $T_{\mu \nu}^{\text {self }}$ should not affect our analysis at higher orders, since $T_{\mu \nu}^{\text {self }}$ enters our equations only in the form $\nabla^{\mu} T_{\mu \nu}^{\text {self }}$. It is not difficult to show that $\nabla^{\mu} T_{\mu \nu}^{\text {self }}$ does have a distributional series with support on $\gamma$ of a form similar to that of $J^{\mu}$ and $T_{\mu \nu}^{M}$.
} 
a Lorentz force trajectory of the external field. Specifically, the charge-current of the body is given by eqs.(29) and (30), the sum of the body stress-energy and the electromagnetic self-field stress-energy is given by eq.(46), and the world line is given by eq.(48). It should be noted from eq.(47) that the electromagnetic self-energy contributes to the mass of the body, i.e., there is a finite "mass renormalization" of the body contributed by its electromagnetic field. It should be emphasized that the description of the body as a point charge/mass is an output of our calculations, that our derivation is mathematically rigorous, and that no infinities have arisen in the derivation of our formula (47) for the mass of the particle.

\section{NEAR-ZONE LIMIT AND PERTURBED MOTION}

We now turn our attention to the first order in $\lambda$ corrections to the motion. In order to do so, we must first address the issue of what we mean by the "corrections to motion". As $\lambda \rightarrow 0$, the body shrinks down to a worldline $\gamma$, so at lowest order, the motion is described by $\gamma$. As shown in the previous section, $\gamma$ must satisfy the Lorentz force equation (48). But, at any $\lambda>0$, the body is of finite size, so in order to find the "correction" to $\gamma$ at finite $\lambda$, we would need to have a "representative worldline" to describe the motion of the body. It would be natural to take this worldline to be the "center of mass". However, as we shall now explain, it is far from obvious as to how to define a "center of mass".

The difficulty arises from the fact that "electromagnetic self-energy" must be included in a definition of center of mass. Indeed, we have already seen in eq.(47) above that, at lowest nonvanishing order, electromagnetic self-energy contributes to the mass of the body. Since energy can be exchanged between the matter and electromagnetic self-field-only their sum, $m$, is conserved at leading order - it clearly would not make sense to omit the electromagnetic self-energy from the definition of the center of mass. However, if one defines the electromagnetic self-stress-energy $T_{\mu \nu}^{\text {self }}$ at finite $\lambda$ to be the electromagnetic stress-energy of the retarded solution $F_{\mu \nu}^{\text {self }}$, then the stress-energy of radiation that was emitted by the body in the distant past would be included. Furthermore, integrals over the self-stressenergy defined this way - corresponding to that stress-energy's contributions to mass, center of mass, etc.-would diverge, on account of the slow, $1 / r$ falloff of the retarded self-field. It is therefore clear on physical and mathematical grounds that the full retarded self-field may not be used to provide a definition of center of mass. On the other hand, it is far from 
obvious as to how one can exclude the energy of the "emitted radiation" while including the energy of the "Coulomb self-field", since one cannot make a clean distinction between the two at finite times.

Recently, one of us [8] has proposed an appropriate definition of the "center of mass" for charged bodies that properly takes into account the body's electromagnetic self-energy while not including radiation emitted in the distant past. It should be possible to use the "representative worldline" provided by this definition at small but finite $\lambda$ to define a notion of the perturbative corrections to $\gamma$ to all orders in $\lambda$. However, in this paper, we shall be concerned only with the leading order correction to $\gamma$. As we shall see below, at this order, there is a very simple and straightforward notion of "center of mass" - including selffield contributions - that can be used to define the perturbed motion, and we will use this notion in our analysis. It can be shown that this notion agrees with the much more general definition of [8] to this order. ${ }^{9}$

Our analysis is most conveniently carried out in the "near zone" picture. Therefore, in subsection IVA we introduce the near-zone limit for the charge-current, stress-energy, electromagnetic field, and metric, and we determine the properties of the various near-zone fields. In subsection IVB, we present our definitions of the body parameters. Finally, in subsection IVC we re-derive the background Lorentz force motion in the near-zone context, and then compute the perturbed motion.

\section{A. Near-zone Limit and Properties}

As analyzed in detail in the previous section, the ordinary ("far-zone") limit associated with our one-parameter family sees the body shrink down in size to an effective point particle description. Here we define a second, "near-zone" limit wherein the body remains at fixed size. To accomplish this, we measure spacetime distances (at $\lambda>0$ ) with a rescaled metric,

$$
\bar{g}_{a b} \equiv \lambda^{-2} g_{a b}
$$

\footnotetext{
${ }^{9}$ It was emphasized in [8] that there is a class of reasonable definitions of generalized momenta and hence center of mass that one may adopt. The differences between the definitions begin at $O\left(\lambda^{2}\right)$ in the perturbation series discussed in this paper, which is precisely the order at which our type of definition would break down.
} 
The inverse metric $\bar{g}^{a b}$ is related by $\bar{g}^{a b}=\lambda^{2} g^{a b}$. The Maxwell and matter equations, however, are not satisfied relative to the barred metric $\bar{g}_{a b}$. Therefore, we also introduce rescaled fields, ${ }^{10}$

$$
\begin{aligned}
\bar{J}^{a} & \equiv \lambda^{3} J^{a} \\
\bar{T}_{a b}^{M} & \equiv T_{a b}^{M} \\
\bar{F}_{a b} & \equiv \lambda^{-1} F_{a b},
\end{aligned}
$$

so that the Maxwell and matter equations,

$$
\begin{aligned}
\bar{\nabla}^{b} \bar{F}_{a b} & =4 \pi \bar{J}_{a} \\
\bar{\nabla}_{[a} \bar{F}_{b] c} & =0 \\
\bar{\nabla}_{a} \bar{J}^{a} & =0 \\
\bar{\nabla}^{b}\left(\bar{T}_{a b}^{B}+\bar{T}_{a b}^{E M}\right) & =0,
\end{aligned}
$$

are satisfied. Here the barred metric $\bar{g}_{a b}$ is used to raise and lower indices, and $\bar{\nabla}_{a}$ is the derivative operator associated with the barred metric (which, however, agrees with the derivative operator associated with the unbarred metric). The electromagnetic stress-energy $\bar{T}_{a b}^{E M}$ is constructed from $\bar{F}_{a b}$ in the usual way.

To complete the description of the near-zone limit, we introduce at each time $t_{0}$ the "scaled coordinates" $\left(\bar{t}, \bar{x}^{i}\right)$ defined by

$$
\bar{t} \equiv \frac{t-t_{0}}{\lambda}, \quad \bar{x}^{i} \equiv \frac{x^{i}-z^{i}\left(t_{0}\right)}{\lambda}
$$

The limit as $\lambda \rightarrow 0$ at fixed $\left(\bar{t}, \bar{x}^{i}\right)$ of a barred quantity in barred coordinates constitutes its near-zone limit. Physically, as $\lambda \rightarrow 0$, an observer at fixed $\left(\bar{t}, \bar{x}^{i}\right)$ approaches the spacetime point $\left(t=t_{0}, x^{i}=z^{i}\left(t_{0}\right)\right)$ along with the shrinking body, but since the observer uses the rescaled metric to measure distances, the space and time intervals between the observer and the point $\left(t_{0}, z^{i}\left(t_{0}\right)\right)$ remain finite. Thus the near-zone limit has the interpretation

${ }^{10}$ Note that these rescalings are modified if indices are raised or lowered, since we use the barred metric to raise and lower indices for barred quantities and the unbarred metric to raise and lower indices for unbarred quantities. For example, we have $\bar{J}_{a}=\lambda J_{a}$. 
of "zooming in" on the body (in space and time) at the same rate at which the body is shrinking.

In order investigate the properties of the near-zone limit, it is convenient to specialize to coordinates where $z^{i}(t)=0$, and we shall do so for the remainder of this paper. In this case, near-zone coordinate components are related to far-zone coordinate components by simple expressions,

$$
\begin{aligned}
\bar{g}_{\bar{\mu} \bar{\nu}}\left(\lambda, t_{0} ; \bar{t}, \bar{x}^{i}\right) & =g_{\mu \nu}\left(\lambda, t=t_{0}+\lambda \bar{t}, x^{i}=\lambda \bar{x}^{i}\right) \\
\bar{J}^{\bar{\mu}}\left(\lambda, t_{0} ; \bar{t}, \bar{x}^{i}\right) & =\lambda^{2} J^{\mu}\left(\lambda, t=t_{0}+\lambda \bar{t}, x^{i}=\lambda \bar{x}^{i}\right) \\
\bar{T}_{\bar{\mu} \bar{\nu}}^{M}\left(\lambda, t_{0} ; \bar{t}, \bar{x}^{i}\right) & =\lambda^{2} T_{\mu \nu}^{M}\left(\lambda, t=t_{0}+\lambda \bar{t}, x^{i}=\lambda \bar{x}^{i}\right) \\
\bar{F}_{\bar{\mu} \bar{\nu}}\left(\lambda, t_{0} ; \bar{t}, \bar{x}^{i}\right) & =\lambda F_{\mu \nu}\left(\lambda, t=t_{0}+\lambda \bar{t}, x^{i}=\lambda \bar{x}^{i}\right) .
\end{aligned}
$$

Note the presence of bars on the indices for the tensors on the left side of these equations (denoting components in barred coordinates) and the absence of bars on the indices of tensors on the right sides (denoting corresponding components in the corresponding unbarred coordinates). It then follows directly from our assumptions and eq. (20) that

$$
\begin{aligned}
\bar{J}^{\bar{\mu}}\left(\lambda, t_{0} ; \bar{t}, \bar{x}^{i}\right) & =\tilde{J}^{\mu}\left(\lambda, t_{0}+\lambda \bar{t}, \bar{x}^{i}\right) \\
\bar{T}_{\bar{\mu} \bar{\nu}}^{M}\left(\lambda, t_{0} ; \bar{t}, \bar{x}^{i}\right) & =\tilde{T}_{\mu \nu}^{M}\left(\lambda, t_{0}+\lambda \bar{t}, \bar{x}^{i}\right) \\
\bar{F}_{\bar{\mu} \bar{\nu}}\left(\lambda, t_{0} ; \bar{t}, \bar{x}^{i}\right) & =\tilde{F}_{\mu \nu}\left(\lambda, t_{0}+\lambda \bar{t}, \bar{x}^{i}\right)+\lambda F_{\mu \nu}^{\operatorname{ext}}\left(\lambda, t_{0}+\lambda \bar{t}, \lambda \bar{x}^{i}\right) .
\end{aligned}
$$

This gives the behavior of the near-zone fields in terms of the (smooth) "tilded" quantities of our assumptions. Equations (62)-(64) make clear that in the near-zone limit the size of the body remains finite (as measured by the barred metric) and all properties of the body (as described by the barred fields) go to well-defined, finite limits. We also see that the effects of the external universe, as well as of the body's own future and past, reduce in importance and finally disappear at $\lambda=0$. This contrasts with the far-zone limit analyzed in the previous section, where the effects of the external universe go to well defined, finite limits, but the effects of the body reduce in importance and finally disappear at $\lambda=0$.

Since the near-zone fields have now been seen explicitly to be smooth in $\lambda, \bar{t}, \bar{x}^{i}$, we know in particular that the near-zone perturbation series exists to all orders in $\lambda$. We will denote the terms in this series in analogy with the notation used in the far-zone limit: 
For a generic barred quantity $\bar{f}$, we write $\bar{f}^{(n)}\left(\bar{x}^{\mu}\right)=\frac{1}{n !} \lim _{\lambda \rightarrow 0} \frac{\partial^{n}}{\partial \lambda^{n}} \bar{f}\left(\lambda, \bar{x}^{\mu}\right)$. Thus it is our convention that the superscript $(n)$ refers to $\lambda$-derivatives at $\lambda=0$ at fixed $\bar{x}^{\mu}$ when the superscripted quantity is barred; otherwise, it refers to $\lambda$-derivatives at fixed $x^{\mu}$. Note that since the charge-current and material stress-energy are of compact support in $\bar{x}^{i}$ at all $\lambda$, this property also holds at each order in perturbation theory.

It follows immediately from eqs.(58)-(61) that each near-zone quantity $\bar{f}$ depends on $t_{0}$ and $\bar{t}$ only in the combination $t_{0}+\lambda \bar{t}$, and that $\bar{f}$ must be a smooth function of $t_{0}+\lambda \bar{t}$. This has two important consequences. First, the dependence of any $n$th order perturbative quantity $\bar{f}^{(n)}$ on $\bar{t}$ can be at most $n$th order polynomial in $\bar{t}$

$$
\bar{f}^{(n)}\left(\bar{t}, \bar{x}^{i}\right)=\sum_{m=0}^{n} A_{m}^{(n)}\left(\bar{x}^{i}\right) \bar{t}^{m}
$$

In particular, all zeroth order near-zone quantities are stationary. Second, we have a simple relationship between $\bar{t}$ and $t_{0}$ derivatives of $\bar{f}^{(n)}$,

$$
\frac{\partial}{\partial \bar{t}} \bar{f}^{(n+1)}=\frac{\partial}{\partial t_{0}} \bar{f}^{(n)}
$$

Equations (65) and (66) are satisfied component-by-component by all the near-zone quantities $\bar{J}^{\bar{\mu}}, \bar{T}_{\bar{\mu} \bar{\nu}}^{M}, \bar{F}_{\bar{\mu} \bar{\nu}}, \bar{g}_{\bar{\mu} \bar{\nu}}$. Equation (66) reflects the fact that the near-zone perturbation series at each time $t_{0}$ are all defined with reference to the single ("far-zone") one-parameter-family. Thus, perturbed near-zone quantities at different scaling times $t_{0}$ cannot be specified independently but instead must be related by equation (66). We refer to this relation here, as in we did for the analogous condition in [9], as a "consistency condition". We will make use of the consistency condition in our calculations, below.

We now turn to the relationship between near-zone and far-zone perturbative quantities. In the previous section, we saw that the far-zone expansion of $J^{\mu}$ was distributional in nature, with the $(n+1)$ st order term, $J^{(n+1) \mu}$, in the expansion being described as a sum of multipoles (i.e., derivatives of $\delta$-functions) up to order $n$. It can be seen that at $t=t_{0}$, for $m \leq n$, the $m$ th order multipole (in the sense of an $m$ th-derivative of a delta function) appearing in $J^{(n+1) \mu}$ contributes to the $m$ th multipole moment (in the sense of a moment 
integral) of $\bar{J}^{(n-m) \bar{\mu}}$ at $\bar{t}=0 .{ }^{11}$ Conversely, the multipole moments of the near-zone $p$ th order charge-current $\bar{J}^{(p) \bar{\mu}}$ correspond to moments appearing at $p$ th and higher order in the far-zone expansion of $J^{\mu}$. This is characteristic of the kind of "mixing of orders" of quantities appearing in far-zone and near-zone expansions. Similar results hold for the near-zone and far-zone expansions of $T_{\mu \nu}^{M}$.

Consider, now, the near-zone external electromagnetic field

$$
\bar{F}_{\bar{\mu} \bar{\nu}}^{\operatorname{ext}}\left(\lambda, t_{0} ; \bar{t}, \bar{x}^{i}\right) \equiv \lambda F_{\mu \nu}^{\mathrm{ext}}\left(\lambda, t_{0}+\lambda \bar{t}, \lambda \bar{x}^{i}\right)
$$

Since $F_{\mu \nu}^{\text {ext }}$ is a smooth function of its arguments, we see that the perturbative expansion of $\bar{F}_{\bar{\mu} \bar{\nu}}^{\text {ext }}$ at order $n$ can depend only polynomially on $\bar{x}^{i}$, with the degree of the polynomial being no higher than $n-1$. Explicitly, the first few terms are given by

$$
\begin{aligned}
& \bar{F}_{\bar{\mu} \bar{\nu}}^{\operatorname{ext},(0)}\left(\lambda, t_{0} ; \bar{t}, \bar{x}^{i}\right)=0 \\
& \bar{F}_{\bar{\mu} \bar{\nu}}^{\operatorname{ext},(1)}\left(\lambda, t_{0} ; \bar{t}, \bar{x}^{i}\right)=\left.F_{\mu \nu}^{\mathrm{ext},(0)}\right|_{t=t_{0}, x^{i}=0} \\
& \bar{F}_{\bar{\mu} \bar{\nu}}^{\operatorname{ext},(2)}\left(\lambda, t_{0} ; \bar{t}, \bar{x}^{i}\right)=\left.F_{\mu \nu}^{\mathrm{ext},(1)}\right|_{t=t_{0}, x^{i}=0}+\left.\bar{x}^{i} \partial_{i} F_{\mu \nu}^{\mathrm{ext},(0)}\right|_{t=t_{0}, x^{i}=0}+\left.\bar{t} \partial_{0} F_{\mu \nu}^{\mathrm{ext},(0)}\right|_{t=t_{0}, x^{i}=0}
\end{aligned}
$$

These equations again show explicitly the characteristic "mixing of orders" in the relationship between near-zone and far-zone fields. For example, equation (69) demonstrates that the first-order near-zone electromagnetic field is a constant (in $\bar{t}, \bar{x}^{i}$ ) whose value is given by the value of the zeroth-order far-zone field on the worldline at time $t_{0}$. Notice also that the zeroth-order near-zone external electromagnetic field vanishes, corresponding to the vanishing effects of the "external universe" in the near-zone limit.

We turn now to the near-zone expansion of the electromagnetic self-field $F_{\mu \nu}^{\text {self }}$. The farzone expansion of $F_{\mu \nu}^{\text {self }}$ was given above in eq. (32). Writing $r=\lambda \bar{r}$ and $t=t_{0}+\lambda \bar{r}$ in the first line of eq. (32), we obtain the corresponding near-zone series (dropping the remainder

${ }^{11}$ Note, however, that the far-zone multipole series was, in effect, defined with $\gamma$ taken to be at the origin of coordinates, whereas near-zone moments will be defined below relative to a $\lambda$-dependent worldline, so the relationship between higher order far-zone and near-zone multipole moments will be complicated. 
terms for convenience)

$$
\begin{aligned}
\bar{F}_{\bar{\mu} \bar{\nu}}^{\text {self }}(\bar{t}, \bar{r}, \theta, \phi) & =\sum_{n=0}^{N} \sum_{m=0}^{M} \lambda^{n} \bar{r}^{n-m-2}\left(\mathcal{F}_{\mu \nu}\right)_{n m}\left(t_{0}+\lambda \bar{t}, \theta, \phi\right) \\
& =\sum_{n=0}^{N} \sum_{m=0}^{M} \sum_{p=0}^{P} \lambda^{n+p} \bar{t}^{p} \bar{r}^{n-m-2}\left(\mathcal{F}_{\mu \nu}\right)_{n m p}\left(t_{0}\right)
\end{aligned}
$$

where we have defined $\left.\left(\mathcal{F}_{\mu \nu}\right)_{n m p} \equiv \frac{1}{p !}\left(\frac{\partial}{\partial t}\right)^{p}\left(\mathcal{F}_{\mu \nu}\right)_{n m}\right|_{t=t_{0}}$ in the second line. Note that this expansion gives useful information at large $\bar{r}$, in contrast to the far-zone expansion eq. (32), which gives useful information at small $r$. (Of course, these series contain precisely the same information, which is useful only at both small $\alpha=r=\lambda \bar{r}$ and small $\beta=\lambda / r=1 / \bar{r}$.) Note that in the near-zone series, at $n$th order in $\lambda$ the highest possible combined positive powers of $\bar{t}$ and $\bar{r}$ is $n$, but arbitrarily high inverse powers of $\bar{r}$ can occur. Comparing with eq. (34), we can explicitly evaluate the first few orders of the near-zone series for $F_{\bar{\mu} \bar{\nu}}^{\text {self }}$ at $\bar{t}=0$ as follows

$$
\begin{aligned}
&\left.\bar{F}_{i 0}^{\text {self, }(0)}\right|_{\bar{t}=0}=\frac{q}{\bar{r}^{2}} n_{i}+O\left(\frac{1}{\bar{r}^{3}}\right) \\
&\left.\bar{F}_{i j}^{\text {self },(0)}\right|_{\bar{t}=0}=O\left(\frac{1}{\bar{r}^{3}}\right) \\
&\left.\bar{F}_{i 0}^{\text {self, }(1)}\right|_{\bar{t}=0}=\frac{q}{\bar{r}}\left(-\frac{1}{2}\left(a_{j} n^{j}\right) n_{i}-\frac{1}{2} a_{i}\right)+O\left(\frac{1}{\bar{r}^{2}}\right) \\
&\left.\bar{F}_{i j}^{\text {self },(1)}\right|_{\bar{t}=0}=O\left(\frac{1}{\bar{r}^{2}}\right) \\
&\left.\bar{F}_{i 0}^{\text {self, }(2)}\right|_{\bar{t}=0}=q\left(\frac{3}{8}\left(a_{j} n^{j}\right)^{2} n_{i}+\frac{3}{4}\left(a_{j} n^{j}\right) a_{i}+\frac{1}{8} a_{j} a^{j} n_{i}+\frac{1}{2} \dot{a}_{0} n_{i}+\frac{2}{3} \dot{a}_{i}\right)+O\left(\frac{1}{\bar{r}}\right) \\
&\left.\bar{F}_{i j}^{\text {self },(2)}\right|_{\bar{t}=0}=-q \frac{1}{2} \dot{a}_{[i} n_{j]}+O\left(\frac{1}{\bar{r}}\right),
\end{aligned}
$$

where, in this equation and all following equations in this paper, we drop the bars on the coordinate components of barred quantities, i.e., it is understood that the components of any barred quantity is taken in barred coordinates. 


\section{B. Center of Mass and Body Parameters}

In order to determine the corrections to Lorentz force motion, we would like to define a notion of the "center of mass worldline" of the body at finite $\lambda$. However, as discussed at the beginning of this section, this is a highly nontrivial notion for a charged body, since it is far from obvious how self-field contributions to the center of mass should be included. Fortunately, in order to define the first order correction to Lorentz force motion in the far zone, we only need to define a notion of center of mass to zeroth order in the near zone. As we shall now see, this can be done in a very simple and straightforward manner. However, if we wished to calculate higher order corrections, we would need to employ a much more sophisticated notion of "center of mass", such as given in [8].

To define the center of mass to first order in $\lambda$, consider an arbitrary smooth, oneparameter family of worldlines $\gamma(\lambda)$ such that $\gamma(0)$ is the worldline $\gamma$ of condition (i) of section II. Choose Fermi normal coordinates about $\gamma(\lambda)$, corresponding to working in the "rest frame" of the body. In these coordinates, the (flat) spacetime metric takes the form (see,e.g.,[13])

$$
\begin{aligned}
& g_{00}=-1-2 a_{i}(\lambda, t) x^{i}-\left(a_{i}(\lambda, t) x^{i}\right)^{2}+O\left(r^{3}\right) \\
& g_{i 0}=O\left(r^{3}\right) \\
& g_{i j}=\delta_{i j}
\end{aligned}
$$

where $a^{i}(\lambda, t)$ is the acceleration of $\gamma(\lambda)$ at time $t$. The components of the corresponding scaled metric are given by

$$
\begin{aligned}
& \bar{g}_{00}=-1-2 \lambda a_{i}\left(t_{0}\right) \bar{x}^{i}-\lambda^{2}\left[2 \dot{a}_{i}\left(t_{0}\right) \bar{x}^{i} \bar{t}+\left(a_{i}\left(t_{0}\right) \bar{x}^{i}\right)^{2}+2 \delta a_{i}\left(t_{0}\right) x^{i}\right]+O\left(\lambda^{3}\right) \\
& \bar{g}_{i 0}=O\left(\lambda^{3}\right) \\
& \bar{g}_{i j}=\delta_{i j}
\end{aligned}
$$

where we have defined $a^{i}(t) \equiv a^{i}(\lambda=0, t)$ and $\delta a^{i} \equiv \partial_{\lambda} a^{i}(\lambda=0, t)$, and the overdot denotes the derivative with respect to $t$. Thus, $a^{i}$ corresponds to the (far-zone) acceleration of the unperturbed worldline $\gamma(0)$, and $\delta a^{i}$ corresponds to the perturbed (far-zone) acceleration of $\gamma(\lambda)$ to first order in $\lambda$. In accordance with the conventions stated at the end of the previous subsection, we have dropped the bars on the coordinate components of $\bar{g}_{\bar{\mu} \bar{\nu}}$ on the left side 
of these equations.

As before, we define the total body stress-energy tensor $\bar{T}_{a b}$ by

$$
\bar{T}_{a b} \equiv \bar{T}_{a b}^{M}+\bar{T}_{a b}^{\text {self }}
$$

At zeroth order in the near-zone, the spacetime metric (80) is Minkowskian and the total body stress-energy is stationary (since all zeroth order near-zone quantities are stationary). Therefore, it is natural to define the total body mass at zeroth order in the near-zone by

$$
m\left(t_{0}\right) \equiv \int \bar{T}_{00}^{(0)} d^{3} \bar{x}
$$

Since $\bar{F}_{\mu \nu}^{\text {self,(0) }}$ is a stationary, regular solution of Maxwell's equations that falls off at large $\bar{r}$ as $1 / \bar{r}^{2}$, the zeroth order self-field stress-energy falls off as $1 / \bar{r}^{4}$, so the integral defining $m$ converges. Indeed, it is easily seen that $m$ agrees with the total body mass at first order in the far-zone, eq. (47), which we also denoted as $m$. Therefore, in particular, $m$ is independent of $t_{0}$, a fact we will confirm in the next subsection by our near-zone analysis.

We define the center of mass, $\bar{X}_{\mathrm{CM}}^{i}$, to zeroth order in the near zone by,

$$
\bar{X}_{\mathrm{CM}}^{i}\left(t_{0}\right)=\frac{1}{m} \int \bar{T}_{00}^{(0)} \bar{x}^{i} d^{3} \bar{x}
$$

The integrand in this equation falls off only as $1 / \bar{r}^{3}$, so the integral defining $\bar{X}_{\mathrm{CM}}^{i}$ does not converge absolutely. However, the $1 / \bar{r}^{4}$ part of $\bar{T}_{00}^{(0)}$ (computed from equation (73)) is spherically symmetric, so the angular average of the $1 / \bar{r}^{3}$ part of the integrand vanishes. Thus, the integral defining $\bar{X}_{\mathrm{CM}}^{i}$ is well defined as a limit as $\bar{R} \rightarrow \infty$ of the integral over a ball of radius $\bar{R}$.

The center of mass changes under a change of the origin of the near-zone coordinates $\bar{x}^{i}$. This corresponds to a first-order in $\lambda$ change of far-zone origin $x^{i}$, or, equivalently, to a change in the choice of $\gamma(\lambda)$ to first-order in $\lambda$ in the far-zone. We therefore may define the perturbed motion to first-order in $\lambda$ in the far-zone by the demand that the zeroth order near-zone center of mass vanish,

$$
\bar{X}_{\mathrm{CM}}^{i}\left(t_{0}\right)=0
$$

Since $\bar{X}_{\mathrm{CM}}^{i}$ is defined relative to the Fermi (i.e., "rest frame") coordinate components of the 
stress-energy, this demand corresponds to the requirement that an observer moving along the worldline assign his own position as the center of mass of the body at each time $t_{0}$. In the next subsection, we shall see by direct computation that this condition gives rise to an equation satisfied by the perturbed acceleration $\delta a^{i}=\left.\partial_{\lambda} a^{i}\right|_{\lambda=0}$, thereby defining a correction to Lorentz force motion.

We define the spin tensor $S^{\mu \nu}$ to zeroth order in the near zone by

$$
S^{0 j}\left(t_{0}\right)=-S^{j 0}\left(t_{0}\right)=\int \bar{T}^{(0) 00} \bar{x}^{j} d^{3} \bar{x}
$$

and

$$
S^{i j}\left(t_{0}\right) \equiv 2 \int \bar{T}_{0}^{(0) i} \bar{x}^{j} d^{3} \bar{x}
$$

Thus, when the center of mass condition is imposed, we have

$$
S^{0 j}=0
$$

We shall assume that the center of mass condition has been imposed in the following. As we shall see from eq.(102) below, it follows from lowest order conservation of stress-energy that $S^{i j}$ is antisymmetric, $S^{i j}=-S^{j i}$. Note that the integral defining $S^{i j}$ converges absolutely, since $\bar{F}_{0 i}^{\text {self,(0) }}$ falls off as $1 / \bar{r}^{2}$ and $\bar{F}_{i j}^{\text {self,(0) }}$ falls off as $1 / \bar{r}^{3}$. The spin vector $S_{i}$ is defined by

$$
S_{i}=\frac{1}{2} \epsilon_{i j k} S^{j k}
$$

To first order in the near-zone, the spacetime metric (80) is no longer Minkowskian, but it is stationary, with timelike Killing field $\partial / \partial \bar{t}$. The stress-energy current $\bar{T}_{\mu \nu}(\partial / \partial \bar{t})^{\nu}$ is therefore conserved to first order, and it is natural to define the first order correction to the mass, $\delta m$, by

$$
\left.\delta m\left(t_{0}\right) \equiv \int_{\Sigma} \frac{\partial}{\partial \lambda}\left(\bar{T}_{a b}\left(\frac{\partial}{\partial \bar{t}}\right)^{b} d \Sigma^{a}\right)\right|_{\lambda=0},
$$

where the integral is independent of the spacelike hypersurface $\Sigma$. This yields

$$
\delta m\left(t_{0}\right)=\left.\int \bar{T}_{00}^{(1)}\right|_{\bar{t}=0} d^{3} \bar{x}
$$

where the center of mass condition has been used. Since the stress-energy $\bar{T}_{00}^{(1)}$ decays only 
as $1 / \bar{r}^{3}$ on account of the "cross term" contribution of $\bar{F}_{\bar{\mu} \bar{\nu}}^{\text {self, }(0)}$ and $\bar{F}_{\bar{\mu} \bar{\nu}}^{\text {self, }(1)}$, this integral does not converge absolutely. However, the explicit asymptotic form of $\bar{T}_{00}^{(1)}$ (computed from equations (73+760) $)$ is

$$
\left.\bar{T}_{00}^{(1)}\right|_{\bar{t}=0}=-\frac{1}{4 \pi} \frac{q^{2}}{\bar{r}^{3}} a_{j} n^{j}+O\left(\frac{1}{\bar{r}^{4}}\right),
$$

from which it can be seen that the angular average of the $1 / \bar{r}^{3}$ part vanishes, so that the integral defining $\delta m$ is well defined as a limit as $\bar{R} \rightarrow \infty$ of the integral over a ball of radius $\bar{R}$. In view of eq. (90), it would be natural to interpret $\delta m$ as the first order correction to the rest mass of the body (including its electromagnetic self-energy).

The total charge $q(\lambda)$ is given at finite $\lambda$ by

$$
q(\lambda)=\int_{\Sigma} J^{a} d \Sigma_{a}
$$

and is independent of the choice of hypersurface $\Sigma$. To zeroth order in the near-zone, the charge $q$ is therefore given by

$$
q \equiv \int \bar{J}^{(0) 0} d^{3} \bar{x}
$$

It is not difficult to see that this agrees with the first order charge in the far-zone, which we previously also denoted as $q$.

The zeroth order electromagnetic dipole moment tensor $Q^{\mu \nu}$ of the body is defined by

$$
Q^{\mu j}\left(t_{0}\right) \equiv \int \bar{J}^{(0) \mu} \bar{x}^{j} d^{3} \bar{x}
$$

together with $Q^{j 0}=-Q^{0 j}$. We shall see in the next subsection that the purely spatial components $Q^{i j}$ are automatically antisymmetric by lowest-order conservation of charge, so we have $Q^{\mu \nu}=Q^{[\mu \nu]}$. The time-space components of $Q^{\mu \nu}$ correspond to the electric dipole moment

$$
p^{i}=Q^{0 i}
$$

whereas the purely spatial components of $Q^{\mu \nu}$ correspond to the magnetic dipole moment

$$
\mu_{i}=-\frac{1}{2} \epsilon_{i j k} Q^{j k} .
$$


By eq.(92), we see that the first order near-zone correction, $\delta q$, to the charge is given by

$$
\left.\delta q \equiv \int \bar{J}^{(1) 0}\right|_{\bar{t}=0} d^{3} \bar{x}+a^{i} Q_{0 i}
$$

Note that the analog of the last term in this equation was absent from eq. (90) on account of the imposition of the center of mass condition. Since $q(\lambda)$ is independent of $t_{0}$ for all $\lambda$, it follows immediately that $\delta q$ is independent of $t_{0}$.

\section{Derivation of Motion}

We now turn to the derivation of the perturbed equations of motion, which will be

obtained directly from conservation of stress-energy, eq. (56). Writing $\bar{T}_{a b}=\bar{T}_{a b}^{M}+\bar{T}_{a b}^{\text {self }}$ as before, we obtain from eq. (56)

$$
\bar{\nabla}^{b} \bar{T}_{a b}=\bar{J}^{b} \bar{F}_{a b}^{\mathrm{ext}}
$$

At zeroth order in the near-zone, the near-zone metric is flat (see eq. (800)), the external electromagnetic field vanishes (see eq. (68)), and all zeroth order near-zone quantities are stationary (see eq. (65)). Therefore, at zeroth order, we obtain

$$
\bar{\partial}^{j} \bar{T}_{\mu j}^{(0)}=0,
$$

where we again remind the reader that we have dropped bars on indices of barred quantities. By multiplying the time and space components of this equation by $x^{i}$ and integrating over space, we obtain

$$
\begin{aligned}
& \int \bar{T}_{i 0}^{(0)} d^{3} \bar{x}=0 \\
& \int \bar{T}_{i j}^{(0)} d^{3} \bar{x}=0
\end{aligned}
$$

Similarly, by multiplying the time and space components by $x^{i} x^{k}$ and integrating over space, 
we obtain

$$
\begin{aligned}
\int \bar{T}_{0}^{(0) i}{ }_{0}^{k} d^{3} \bar{x} & =-\int \bar{T}_{0}^{(0) k} \bar{x}^{i} d^{3} \bar{x} \\
\int \bar{T}_{i j}^{(0)} \bar{x}^{k} d^{3} \bar{x} & =0 .
\end{aligned}
$$

Equation (102) demonstrates the previously claimed anti-symmetry of the (lowest-order) spin of the body, defined by eq. (86). Equation (103) follows from an analogous antisymmetry in $i$ and $k$, combined with the symmetry of $T_{i j}$ in $i$ and $j$.

At zeroth order in the near-zone, conservation of charge-current yields

$$
\bar{\partial}_{i} \bar{J}^{(0) i}=0
$$

from which we can analogously derive the useful relations,

$$
\begin{aligned}
\int \bar{J}^{(0) i} d^{3} \bar{x} & =0 \\
\int \bar{J}^{(0) i} \bar{x}^{j} d^{3} \bar{x} & =-\int \bar{J}^{(0) j} \bar{x}^{i} .
\end{aligned}
$$

The second of these equations demonstrates the previously claimed anti-symmetry of the spatial components of the electromagnetic dipole tensor $Q^{\mu \nu}$ defined by eq.(94).

At first-order in the near-zone, we see the first appearances of the acceleration (via the metric components (80)) and the external field (via equation (69)). At first order, conservation of stress-energy, eq.(98), explicitly yields

$$
\begin{array}{r}
\bar{\partial}^{0} \bar{T}_{00}^{(1)}+\bar{\partial}^{i} \bar{T}_{0 i}^{(1)}+a^{i} \bar{T}_{0 i}^{(0)}-\bar{J}^{(0) \mu} F_{0 \mu}^{\mathrm{ext}}=0 \\
\bar{\partial}^{0} \bar{T}_{k 0}^{(1)}+\bar{\partial}^{j} \bar{T}_{k j}^{(1)}+a_{k} \bar{T}_{00}^{(0)}+a^{j} \bar{T}_{k j}^{(0)}-\bar{J}^{(0) \mu} F_{k \mu}^{\mathrm{ext}}=0
\end{array}
$$

where $a_{i}$ is evaluated at time $t=t_{0}$, and we have used eq. (69) to replace $\bar{F}_{\mu \nu}^{\text {ext,(1) by }}$ $\left.F_{\mu \nu}^{\mathrm{ext}} \equiv F_{\mu \nu}^{\mathrm{ext},(0)}\right|_{t=t_{0}, x^{i}=0}$.

We shall now use these equations to derive evolution equations for the mass and spin to lowest order, as well as to determine the lowest order motion. For the evolution of mass, we integrate equation (107) over space (with volume element $d^{3} \bar{x}$ ). Using the consistency condition (66), the first term evaluates to $-d m\left(t_{0}\right) / d t_{0}$. The second term vanishes by inte- 
gration by parts, since $\bar{T}_{0 i}^{(1)}$ falls off like $1 / \bar{r}^{4}$. The third term vanishes by eq. (100), and the fourth term vanishes by eq.(105). Thus we obtain

$$
\frac{d}{d t_{0}} m=0
$$

in agreement with what we already found in our far-zone analysis given in section III.

For the evolution of spin, we multiply eq. (108) by $\bar{x}^{i}$ and integrate over space. Again by the consistency condition the first term evaluates to $-(1 / 2) d S_{i k}\left(t_{0}\right) / d t_{0}$. The third term vanishes by the center of mass condition (184), and the fourth term vanishes by eq. (103). However, the second and fifth terms do not vanish, yielding

$$
\frac{1}{2} \frac{d}{d t_{0}} S_{i k}=\int \bar{T}_{i k}^{(1)} d^{3} \bar{x}+Q_{i}^{\mu} F_{k \mu}^{\text {ext }}
$$

The antisymmetric part of this equation provides the desired evolution equation for the spin,

$$
\frac{d}{d t_{0}} S_{i j}=2 Q_{[i}^{\mu} F_{j] \mu}^{\mathrm{ext}}
$$

The right side of this equation corresponds to the usual formula for the torque on an electromagnetic dipole. It also should be noted that, since we work in Fermi normal coordinates, $d S_{i k} / d t_{0}$ corresponds to the "Fermi derivative" of $S_{i k}$, so eq. (111) automatically includes the "Thomas precession" (see eq.(135) below). The symmetric part of (110) yields the useful relation

$$
\int \bar{T}_{i j}^{(1)} d^{3} \bar{x}=Q_{(i}^{\mu} F_{j) \mu}^{\mathrm{ext}}
$$

To determine the lowest order motion, we integrate equation (108) over space. The first term vanishes by the consistency condition and eq. (100), the second term vanishes since $\bar{T}_{i j}^{(1)}$ falls off as $1 / \bar{r}^{3}$, and the fourth term vanishes by eq. (103). The third and fifth terms yield,

$$
m a_{i}=q F_{i 0}^{\mathrm{ext}}
$$

Thus, we reproduce the Lorentz force law previously derived by the far-zone analysis of section III.

Finally, we derive an additional relation from first-order stress-energy conservation by multiplying eq. (107) by $\bar{x}^{i}$ and integrating over space. The first term vanishes by the 
consistency condition (66) and the center of mass condition (84). The second term may be integrated by parts; the associated surface term vanishes because $\bar{T}_{0 i}^{(1)}$ falls off as $1 / \bar{r}^{4}$. The third and fourth terms are simply expressed in terms of the spin (86) and electromagnetic dipole (94) tensors. We obtain

$$
\int \bar{T}^{(1) j}{ }_{0} d^{3} \bar{x}=\frac{1}{2} a_{i} S^{i j}+Q^{i j} F_{i 0}^{\mathrm{ext}}
$$

In a similar manner, at first-order in the near-zone, conservation of charge-current yields

$$
\bar{\partial}_{0} \bar{J}^{(1) 0}+\bar{\partial}_{j} \bar{J}^{(1) j}+a_{j}^{(0)} \bar{J}^{(1) j}=0
$$

Multiplying by $\bar{x}^{i}$ and integrating over space, we obtain

$$
\int \bar{J}^{(1) i} d^{3} \bar{x}=\frac{d}{d t_{0}} Q^{0 i}+a_{j} Q^{j i}
$$

We now shall derive an equation for the perturbed acceleration $\delta a^{i}$ (see eq. (80)), as well as an evolution equation for the perturbed mass, $\delta m$ (see eq. (90)). At second-order, conservation of stress-energy yields

$$
\begin{aligned}
0 & =\bar{\partial}^{0} \bar{T}_{00}^{(2)}+\bar{\partial}^{i} \bar{T}_{0 i}^{(2)}+a^{i} T_{0 i}^{(1)}+\delta a^{i} T_{0 i}^{(0)}+\dot{a}^{i} \bar{T}_{0 i}^{(0)} \bar{t}+2 a_{i} \bar{x}^{i} \bar{\partial}_{0} \bar{T}_{00}^{(1)}+2 \dot{a}_{i} \bar{x}^{i} \bar{T}_{00}^{(0)}-a^{i} \bar{x}^{i} a^{j} \bar{T}_{0 j}^{(0)} \\
& -\bar{J}^{(0) \mu}\left(\bar{x}^{i} \partial_{i} F_{0 \mu}^{\mathrm{ext}}+\dot{F}_{0 \mu}^{\mathrm{ext}} \bar{t}+\delta F_{0 \mu}^{\mathrm{ext}}\right)-\bar{J}^{(1) \mu} F_{0 \mu}^{\mathrm{ext}} \\
0 & =\bar{\partial}_{0} \bar{T}_{k 0}^{(2)}+\bar{\partial}^{i} \bar{T}_{k i}^{(2)}+a_{k} \bar{T}_{00}^{(1)}+a^{i} \bar{T}_{k i}^{(1)}+\delta a_{k} \bar{T}_{00}^{(0)}+\delta a^{i} \bar{T}_{k i}^{(0)}+\dot{a}_{k} \bar{T}_{00}^{(1)} \bar{t}+\dot{a}^{i} \bar{T}_{k i}^{(1)} \bar{t} \\
& +2 a_{i} \bar{x}^{i} \bar{\partial}_{0} \bar{T}_{k 0}^{(1)}+\dot{a}_{i} \bar{x}^{i} T_{k 0}^{(0)}-a_{i} \bar{x}^{i} a^{j} \bar{T}_{k j}^{(0)}-3 a_{k} a_{i} \bar{x}^{i} \bar{T}_{00}^{(0)} \\
& -\bar{J}^{(0) \mu}\left(\bar{x}^{i} \partial_{i} F_{k \mu}^{\mathrm{ext}}+\dot{F}_{k \mu}^{\mathrm{ext}} \bar{t}+\delta F_{k \mu}^{\mathrm{ext}}\right)-\bar{J}^{(1) \mu} F_{k \mu}^{\mathrm{ext}}
\end{aligned}
$$

where we have used eqs. (80) and (70) and have written $\left.\delta F_{\mu \nu}^{\mathrm{ext}} \equiv F_{\mu \nu}^{\mathrm{ext},(1)}\right|_{t=t_{0}, x^{i}=0}$. To derive an equation for the perturbed acceleration $\delta a^{i}$, we evaluate (118) at $\bar{t}=0$ and integrate over space, applying the consistency condition (66) to various terms, using the center of mass condition (84), using eqs. (101) and (103), and using the definitions of the spin and dipole 
tensors given in section IVB. We obtain

$$
\begin{aligned}
0 & =\frac{d}{d t_{0}} \int \bar{T}_{0 k}^{(1)} d^{3} \bar{x}+\int \bar{r}^{2} n^{i} \bar{T}_{k i}^{(2)} d \Omega+a_{k} \delta m+a^{i} \int T_{k i}^{(1)} d^{3} \bar{x}+\delta a_{k} m+a^{i} \frac{d}{d t_{0}} S_{k i}+\frac{1}{2} \dot{a}^{i} S_{k i} \\
& -Q^{\mu i} \partial_{i} F_{k \mu}^{\mathrm{ext}}-\delta F_{k \mu}^{\mathrm{ext}} \int \bar{J}^{(0) \mu} d^{3} \bar{x}-\int \bar{J}^{(1) \mu} F_{k \mu}^{\mathrm{ext}} d^{3} \bar{x}
\end{aligned}
$$

The second term in this equation is the surface term arising from integration by parts of the second term in eq. (118). To evaluate this term, we compute the asymptotic form of $\bar{T}_{i j}^{(2)}$ from equations (73,78), obtaining

$$
\begin{gathered}
\left.\bar{T}_{i j}^{(2)}\right|_{\bar{t}=0}=-\frac{1}{4 \pi} \frac{q^{2}}{\bar{r}^{2}}\left[n_{i} n_{j}\left(6\left(a_{k} n^{k}\right)^{2}+\frac{1}{4} a_{k} a^{k}+\dot{a}_{0}\right)+\delta_{i j}\left(4\left(a_{k} n^{k}\right)^{2}+\frac{1}{4} a_{k} a^{k}+\frac{1}{2} \dot{a}_{0}+\frac{2}{3} \dot{a}_{k} n^{k}\right)\right. \\
\left.\left.\left.+4 a_{k} n^{k} a_{(i} n_{j}\right)+\frac{4}{3} \dot{a}_{(i} n_{j}\right)+\frac{1}{4} a_{i} a_{j}\right]+O\left(\frac{1}{\bar{r}^{3}}\right) .
\end{gathered}
$$

Since the integral over a sphere of the product of an odd number of normal vectors, $n^{i}$, vanishes, only the terms in $\bar{T}_{i j}^{(2)}$ that are proportional to $\dot{a}^{i}$ survive when computing the second term in eq. (119). We obtain

$$
\int \bar{r}^{2} n^{i} \bar{T}_{k i}^{(2)} d \Omega=\frac{2}{3} q^{2} \dot{a}^{i}
$$

which can be recognized as the Abraham-Lorentz-Dirac self-force term (see eq. (9) above).

Returning to eq. (119), we evaluate the first term using eq. (114), we evaluate the fourth term using eq. (112), and we use eqs. (105), (97), and (116) to re-express the final two terms in terms of the electromagnetic dipole and the corrected charge. After some algebra, we obtain

$$
m \delta a_{i}=-(\delta m) a_{i}+(\delta q) F_{i 0}^{\mathrm{ext}}+q \delta F_{i 0}^{\mathrm{ext}}+\frac{2}{3} q^{2} \dot{a}_{i}-\frac{1}{2} Q^{\mu \nu} \partial_{i} F_{\mu \nu}^{\mathrm{ext}}+\frac{d}{d t_{0}}\left(a^{j} S_{j i}-2 Q_{[i}^{j} F_{0] j}^{\mathrm{ext}}\right)
$$

where we have used Maxwell's equation $\partial_{[\mu} F_{\nu \rho]}^{\text {ext }}=0$ to reexpress the fifth term on the right side.

We also can derive an evolution equation for the perturbed mass, $\delta m$, by integrating equation (117) (evaluated at $\bar{t}=0$ ) over $\bar{x}^{i}$, and performing similar manipulations as above. 
The result is,

$$
\frac{d}{d t_{0}} \delta m=\frac{1}{2} Q^{\mu \nu} \partial_{0} F_{\mu \nu}^{\mathrm{ext}}-\frac{d}{d t_{0}}\left(Q^{\mu 0} F_{\mu 0}^{\mathrm{ext}}\right)
$$

Equation (122) is our desired equation for the perturbed acceleration $\delta a^{i}$. As described at the beginning of the next section, this equation can be written as an evolution equation for the deviation vector, $X^{a}$, describing the perturbation from Lorentz force motion. Appearing on the right side of eq. (122) are the lowest order mass, $m$, the lowest order charge, $q$, the lowest order acceleration $a^{i}$, the perturbed mass, $\delta m$, the perturbed charge, $\delta q$, the spin tensor, $S^{\mu \nu}$, and the electromagnetic dipole tensor, $Q^{\mu \nu}$. As previously derived, $m, q$, and $\delta q$ are conserved, and $a^{i}$ is given by the Lorentz force equation (113). The evolution of $S^{\mu \nu}$ is given by eq. (111), and the evolution of $\delta m$ is given by eq. (123). However, we cannot obtain an evolution equation for the electromagnetic dipole tensor $Q^{\mu \nu}$ because the behavior of this quantity is "non-universal". Indeed, it seems clear that by use of a "Maxwell demon" ${ }^{12}$, we could make $Q^{\mu \nu}$ evolve with time any way that we wish. Therefore, additional conditions/assumptions beyond the Maxwell and matter equations (1)-(3) and (7) would need to be adjoined to the equations we have derived in order to get deterministic evolution at this order. In appendix B we provide an example of a system with deterministic evolution by considering the case of a body with no electric dipole moment and a magnetic dipole moment proportional to spin.

We can rewrite our equations of motion in covariant form as follows. In Fermi coordinates based on the worldline $\gamma$, the time direction $(\partial / \partial t)^{a}$ on $\gamma$ coincides with the unit tangent, $u^{a}$ to $\gamma$, so for example, $F_{i 0}^{\text {ext }}$ can be replaced by $u^{a} F_{i a}^{\text {ext }}$. Similarly, spatial components of quantities on $\gamma$ correspond to projections orthogonal to $u^{a}$, so, for example, the spatial components $u^{a} F_{i a}^{\text {ext }}$ correspond to the spacetime tensor $\left(\delta^{c}{ }_{b}+u^{c} u_{b}\right) u^{a} F_{c a}^{\text {ext }}=u^{a} F_{b a}^{\text {ext }}$. The spatial derivative, $\partial_{i}$, in Fermi coordinates can be expressed in terms of the spatial projection of the covariant derivative $\nabla_{\mu}$ by using the following formula for the Christoffel symbol in Fermi coordinates,

$$
\left.\Gamma_{\mu \nu}^{\alpha}\right|_{\gamma}=a^{\alpha} u_{\mu} u_{\nu}-u^{\alpha} u_{\mu} a_{\nu}-u^{\alpha} a_{\mu} u_{\nu}
$$

Thus, $\partial_{i} F_{\mu \nu}^{\text {ext }}$ corresponds to $\left(\delta^{d}{ }_{c}+u^{d} u_{c}\right) \nabla_{d} F_{a b}^{\text {ext }}+2 a_{c} u^{e} F_{e[b} u_{a]}$. Finally, since, by construction, the Fermi coordinate vector fields $\left\{(\partial / \partial t)^{a},\left(\partial / \partial x^{i}\right)^{a}\right\}$ are Fermi transported along $\gamma$, it is

\footnotetext{
${ }^{12}$ Of course, the stress-energy of the Maxwell demon must be included in $T_{\mu \nu}^{M}$.
} 
clear that taking the time derivative, " $d / d t_{0}$ ", of Fermi coordinate components along $\gamma$ corresponds to taking the Fermi derivative of the corresponding tensors along $\gamma$. Here the Fermi derivative, $D_{F} / d \tau$, is given by the usual covariant derivative $D / d \tau \equiv u^{a} \nabla_{a}$ along $\gamma$ combined with a Lorentz boost in the plane defined by $u^{a}$ and $a^{a}$. Specifically, for a scalar function $f$ on $\gamma$ we have

$$
\frac{D_{F}}{d \tau} f=\frac{D}{d \tau} f \equiv u^{a} \nabla_{a} f
$$

for a covector field $f_{a}$ on $\gamma$ we have

$$
\frac{D_{F}}{d \tau} f_{a}=\frac{D}{d \tau} f_{a}+2 f^{c} a_{[a} u_{c]}
$$

and for a tensor field $f_{a b}$ on $\gamma$ we have

$$
\frac{D_{F}}{d \tau} f_{a b}=\frac{D}{d \tau} f_{a b}+2 f_{a}^{c} a_{[b} u_{c]}+2 f_{b}^{c} a_{[a} u_{c]}
$$

In covariant notation, the evolution equations become

$$
\begin{aligned}
m \delta a_{a}= & -a_{a} \delta m+\delta\left[q F_{a b}^{\mathrm{ext}} u^{b}\right]+a_{a} u_{b} u^{c} Q^{b d} F_{c d}^{\mathrm{ext}} \\
& +\left(g_{a}^{b}+u_{a} u^{b}\right)\left\{\frac{2}{3} q^{2} \frac{D_{F}}{d \tau} a_{b}-\frac{1}{2} Q^{c d} \nabla_{b} F_{c d}^{\mathrm{ext}}+\frac{D_{F}}{d \tau}\left(a^{c} S_{c b}-2 u^{d} Q_{[b}^{c} F_{d] c}^{\mathrm{ext}}\right)\right\} \\
\frac{D_{F}}{d \tau} S_{a b}= & 2\left(g_{a}^{c}+u^{c} u_{a}\right)\left(g_{b}^{d}+u^{d} u_{b}\right) Q_{[c}^{e} F_{d] e}^{\mathrm{ext}} \\
\frac{D_{F}}{d \tau} \delta m= & \frac{1}{2} Q^{a b} \frac{D_{F}}{d \tau} F_{a b}^{\mathrm{ext}}+\frac{D_{F}}{d \tau}\left(u_{b} u^{c} Q^{b d} F_{c d}^{\mathrm{ext}}\right) .
\end{aligned}
$$

In addition, we have $u^{a} S_{a b}=0$ (see eq. (87) above). We remind the reader that these equations would have to be supplemented with a rule for the evolution of the electromagnetic dipole (arising from additional assumptions about the body) in order to comprise a "closed," deterministic system.

By inspection, we see that eqs. (128) and (130) simplify if we redefine the perturbed mass by

$$
\delta m \rightarrow \delta \hat{m} \equiv \delta m-u_{b} u^{c} Q^{b d} F_{c d}^{\mathrm{ext}}
$$

This corresponds to adding the standard expression, $-\vec{p} \cdot \vec{E}^{\text {ext }}$, for electric dipole interaction energy (with $\vec{E}^{\text {ext }}$ the external electric field in the rest frame of the body) to the definition 
of perturbed mass. Note, however, that we do not make any similar $-\vec{\mu} \cdot \vec{B}$ ext adjustment to the definition of perturbed mass. With this re-definition, eqs. (128) and (130) become

$$
\begin{aligned}
m \delta a_{a}=-a_{a} \delta \hat{m}+\delta\left[q F_{a b}^{\mathrm{ext}} u^{b}\right]+\left(g_{a}{ }^{b}+u_{a} u^{b}\right) & \left\{\frac{2}{3} q^{2} \frac{D_{F}}{d \tau} a_{b}-\frac{1}{2} Q^{c d} \nabla_{b} F_{c d}^{\mathrm{ext}}+\frac{D_{F}}{d \tau}\left(a^{c} S_{c b}-2 u^{d} Q_{[b}^{c} F_{d] c}^{\mathrm{ext}}\right)\right\} \\
\frac{D_{F}}{d \tau} \delta \hat{m} & =\frac{1}{2} Q^{a b} \frac{D_{F}}{d \tau} F_{a b}^{\mathrm{ext}}
\end{aligned}
$$

We finally rewrite eqs. (132), (129) and (133) in terms of the usual covariant derivative $D / d \tau=u^{a} \nabla_{a}$ along $\gamma$, obtaining

$$
\begin{aligned}
\delta\left[\hat{m} a_{a}\right] & =\delta\left[q F_{a b}^{\mathrm{ext}} u^{b}\right]+\left(g_{a}{ }^{b}+u_{a} u^{b}\right)\left\{\frac{2}{3} q^{2} \frac{D}{d \tau} a_{b}-\frac{1}{2} Q^{c d} \nabla_{b} F_{c d}^{\mathrm{ext}}+\frac{D}{d \tau}\left(a^{c} S_{c b}-2 u^{d} Q_{[b}^{c} F_{d] c}^{\mathrm{ext}}\right)\right\} \\
\frac{D}{d \tau} S_{a b} & =2\left(g_{a}{ }^{c}+u_{a} u^{c}\right)\left(g_{b}{ }^{d}+u_{b} u^{d}\right) Q_{[c}^{e} F_{d] e}^{\mathrm{ext}}-2 a^{c} S_{c[a} u_{b]} \\
\frac{D}{d \tau} \delta \hat{m} & =\frac{1}{2} Q^{a b} \frac{D}{d \tau} F_{a b}^{\mathrm{ext}}+2 Q_{a}{ }^{b} F_{b c}^{\mathrm{ext}} a^{[c} u^{a]}
\end{aligned}
$$

where $\delta\left[\hat{m} a_{a}\right] \equiv m \delta a_{a}+(\delta \hat{m}) a_{a}$.

The first term on the right side of equation (134) is simply the corrected Lorentz force. The first term in curly brackets is the Abraham-Lorentz-Dirac self force (see eq. (9) above). The second term in curly brackets corresponds to the usual dipole forces familiar from electrostatics and magnetostatics. The final terms in curly brackets are not usually considered in elementary treatments of electromagnetic forces. We will show elsewhere [15] that these terms are responsible for producing behavior in an orbiting charged body with spin and a magnetic dipole moment that is analogous to behavior found for spinning black holes in binary orbits in general relativity. The first term on the right side of eq. (135) corresponds to the usual dipole torques familiar from electrostatics and magnetostatics. The second term is the Thomas precession. Finally, we note that the (modified) perturbed mass $\delta \hat{m}$ is not constant in time. However, in some cases - for example in the case discussed in appendix B there may be an alternative notion of mass that is conserved.

Equations (134)-(136) together with $u^{a} S_{a b}=0$ provide the complete description of the first-order deviation from Lorentz force motion, eq.(48), that can be derived from Maxwell's equations and conservation of stress-energy alone, and comprise the main result of this paper. 


\section{SELF-CONSISTENT MOTION}

There are no mathematical difficulties that arise if one uses eqs (134)-(136) to obtain the lowest order deviation from Lorentz force motion, eq. (48). The lowest order quantities $m$, $q$, and $F_{a b}^{\text {ext }}$ are to be viewed as "given", and the unperturbed worldline, $\gamma$, and its associated quantities $u^{a}, a^{a}$, and $D a^{a} / d \tau$ are to be viewed as having been determined by solving eq. (48). We wish to determine the perturbed worldline as well as the time evolution of the quantities $S_{a b}, Q_{a b}, \delta q$, and $\delta \hat{m}$. The perturbed worldline is described by a deviation vector, $X^{a}$, on the background worldline $\gamma$, defined as follows: Consider the $\lambda$-dependent family of curves, $\gamma(\lambda)$, introduced in subsection IVB. Parameterize each curve by proper time $\tau$. In arbitrary fixed (i.e., $\lambda$-independent) coordinates $x^{\mu}$, the family of curves is described by $Z^{\mu}(\lambda ; \tau)$. Then the coordinate components of the deviation vector are given by $\left.X^{\mu} \equiv\left(\partial Z^{\mu}(\lambda ; \tau) / \partial \lambda\right)\right|_{\lambda=0}$. It follows that

$$
\delta u^{a}=\frac{D}{d \tau} X^{a}
$$

and

$$
\delta a^{a}=\frac{D}{d \tau} \delta u^{a}=\frac{D^{2}}{d \tau^{2}} X^{a} .
$$

Furthermore, since $\delta F_{a b}^{\text {ext }}=\left.\left(\frac{\partial}{\partial \lambda}\left(\left.F_{a b}^{\operatorname{ext}}(\lambda)\right|_{\gamma(\lambda)}\right)\right)\right|_{\lambda=0}$ we have

$$
\delta F_{a b}^{\mathrm{ext}}=X^{c} \nabla_{c} F_{a b}^{\mathrm{ext}}+F_{a b}^{\mathrm{ext},(1)}
$$

where $F_{a b}^{\text {ext,(1) }}$ is the first order perturbation of the external field arising in far-zone perturbation theory, evaluated on $\gamma$. Again $F_{a b}^{\text {ext,(1) }}$ should be viewed as "given" (and, indeed, it would normally be assumed to vanish). It can then be seen explicitly that eqs. (134)-(136) together with eq. (137) and $D \delta q / d \tau=0$ comprise a system of linear, first-order ordinary differential equations for $\left(X^{a}, \delta u^{a}, S_{a b}, Q_{a b}, \delta q, \delta \hat{m}\right)$. If supplemented by an appropriate evolution equation for $Q_{a b}$ these equations have a unique solution for any given initial values of these quantities. The initial $S_{a b}$ also must satisfy the center of mass condition $S_{a b} u^{a}=0$; this condition on $S_{a b}$ is then preserved by the evolution equation (135). The solutions to these equations are not plagued by the type of pathologies that occur for solutions to the Abraham-Lorentz-Dirac equation (9).

Nevertheless, the above equations do not give a fully satisfactory description of the devi- 
ation from Lorentz force motion due to self-force, spin, and electromagnetic dipole moment. Even if all of the terms on the right side of eq. (134) are small compared to the Lorentz force at all times, the cumulative effects of these terms over time should eventually make the deviation vector, $X^{a}$, become large, at which point the description of motion as a linear perturbation of a single, fixed Lorentz force trajectory cannot be accurate. The solution to this difficulty is not to go to higher order in perturbation theory but to realize that although the deviation from a single Lorentz force trajectory may eventually become large at late times, the local deviation from some Lorentz force trajectory should be small at all times. As discussed in much more depth in [9], to implement the description of motion as a locally small deviation from some (varying) Lorentz force trajectory, we need to find a system of "self-consistent perturbative equations" that satisfies the following criteria: (1) They must have a well posed initial value formulation. (2) They must have the same number of degrees of freedom as the first order perturbative system, so that a correspondence can be made between initial data for the self-consistent perturbative equation and the first order perturbative system. (3) For corresponding initial data, the solutions to the self-consistent perturbative equation should be close to the corresponding solutions of the first order perturbative system over the time interval for which the first order perturbative description should be accurate.

An obvious first attempt to find such a self-consistent perturbative equation would be to simply "delete the $\delta$ 's" from eqs (134) -(136), i.e., take the corrected equations of motion to be

$$
\begin{aligned}
\hat{m} a_{a} & =q F_{a b}^{\mathrm{ext}} u^{b}+\left(g_{a}{ }^{b}+u_{a} u^{b}\right)\left\{\frac{2}{3} q^{2} \frac{D}{d \tau} a_{b}-\frac{1}{2} Q^{c d} \nabla_{b} F_{c d}^{\mathrm{ext}}+\frac{D}{d \tau}\left(a^{c} S_{c b}-2 u^{d} Q_{[b}^{c} F_{d] c}^{\mathrm{ext}}\right)\right\} \\
\frac{D}{d \tau} S_{a b} & =2\left(g_{a}{ }^{c}+u_{a} u^{c}\right)\left(g_{b}{ }^{d}+u_{b} u^{d}\right) Q_{[c}^{e} F_{d] e}^{\mathrm{ext}}-2 a^{c} S_{c[a} u_{b]} \\
\frac{D}{d \tau} \hat{m} & =\frac{1}{2} Q^{a b} \frac{D}{d \tau} F_{a b}^{\mathrm{ext}}+2 Q_{a}{ }^{b} F_{b c}^{\mathrm{ext}} a^{[c} u^{a]}
\end{aligned}
$$

together with $u^{a} S_{a b}=0$. In a sense, this is the simplest modification we can make to the Lorentz force equation (48) that takes into account the first-order perturbative effects of eqs. (134)-(136). However, it is easily seen that although eqs. (140)-(142) are ODEs and thus have an initial value formulation, these equations violate criteria (2) and (3) above. 
Specifically, the role of the acceleration, $a^{a}$, changes from that of a zeroth order "background" quantity in eq. (134) to that of an unknown dynamical variable in eq. (140). Consequently, the terms involving $D a^{a} / d \tau$ on the right side of eq. (140) make this equation be of higher differential order than the corresponding perturbative equation (134). Thus, more initial data is required for eqs (140)-(142) than for eqs (134)-(136), in violation of criterion (2). Criterion (3) also is violated. Indeed, when $Q^{a b}$, and $S_{a b}$ vanish, eq. (140) reduces to the ALD equation (9), so even in this simple case, eqs (140)-(142) have solutions that differ drastically from eqs (134)-(136). It is also worth noting that even when $q$ and $Q^{a b}$ vanish (so there are no electromagnetic effects), if $S_{a b}$ is nonvanishing, eq. (140) is still of higher differential order than eq. (134) on account of the $D\left(a^{a} S_{a b}\right) / d \tau$ term. The resulting unphysical degrees of freedom of the system eqs (140)-(142) together with $u^{a} S_{a b}=0$ give rise to solutions with "helical motions" in addition to the expected solutions with inertial motion.

However, this difficulty can be overcome by the reduction of order procedure described in the Introduction. We replace $D a^{a} / d \tau$ in the two terms in which it appears on the right side of eq. (140) by the right side of eq. (11), and we replace $a^{a}$ on the right sides of eqs. (140)-(142) by the right side of eq. (10). The resulting system of equations - which we will not write out explicitly here - still corresponds to Lorentz force motion corrected by the perturbative effects of eqs. (134)-(136), but the resulting equations now have highest derivative terms of exactly the same form as eqs. (134)-(136). Consequently, criterion (2) holds, and we believe that criterion (3) also holds. Thus, we believe that the reduced order form of eqs (140)-(142) supplemented by $u^{a} S_{a b}=0$ comprise a fully satisfactory system of self-consistent perturbative equations that provide a description of motion that takes into account the lowest order effects of self-force, spin, and electromagnetic dipole moments. For comparison with what can be found in textbooks on electromagnetism, we note that in the non-relativistic limit ${ }^{13}$ and in ordinary vector notation, eq. (140) takes the form

$$
\begin{gathered}
\vec{F}=q(\vec{E}+\vec{v} \times \vec{B})+\frac{2}{3} q^{2} \frac{d \vec{a}}{d t}+p_{i} \vec{\nabla} E^{i}+\mu_{i} \vec{\nabla} B^{i} \\
+\frac{d}{d t}(\vec{S} \times \vec{a}-\vec{\mu} \times \vec{E}-\vec{p} \times \vec{B})
\end{gathered}
$$

\footnotetext{
13 To obtain this equation, we drop all terms in eq. (140) that are quadratic or higher order in velocity, $\vec{v}$, as well as terms linear in $\vec{v}$ that are multiplied by $q^{2}, \vec{p}, \vec{\mu}$ or $\vec{S}$.
} 
where we have written $\vec{F}=m \vec{a}$ and it should again be understood that the terms in $\vec{a}$ and $d \vec{a} / d t$ on the right side of this equation should be eliminated by reduction of order.

Finally, as discussed in [9], there is no reason to expect self-consistent perturbative equations satisfying criteria (1)-(3) to be unique. Although the reduced order form of eqs (140)(142) supplemented by $u^{a} S_{a b}=0$ appears to be a fully satisfactory system of self-consistent perturbative equations corresponding to the corrections to Lorentz force given by eqs (134)(136), other choices of self-consistent perturbative equations are possible. In particular, the following system of equations arises naturally from the analysis of the motion of extended bodies [8]: Define the "force" $f_{a}$ and "torque" $n_{a b}=n_{[a b]}$ in terms of the 4-momentum $P^{a}$ and and electromagnetic dipole $Q^{a b}$ by

$$
\begin{gathered}
f_{a}=\frac{2}{3}(q / M)^{3} h_{a}{ }^{b} P^{d}\left(P^{c} \nabla_{d} F_{b c}^{\mathrm{ext}}-q g^{c f} F_{b c}^{\mathrm{ext}} F_{d f}^{\mathrm{ext}}\right)-\frac{1}{2} Q^{b c} \nabla_{a} F_{b c}^{\mathrm{ext}} \\
n_{a b}=2 Q^{c}{ }_{[a} F_{b] c}^{\mathrm{ext}}
\end{gathered}
$$

where

$$
M \equiv \sqrt{-P_{a} P^{a}}
$$

and

$$
h_{a b} \equiv g_{a b}+P_{a} P_{b} / M^{2}
$$

(The first term in eq. (144) can be recognized as a reduced order form of the AbrahamLorentz-Dirac force.) Then the tangent, $\dot{\gamma}^{a}$, to the center-of-mass worldline (normalized so that $\left.P_{a} \dot{\gamma}^{a}=-M\right)$ is given in terms of $P^{a}, Q^{a b}$ and the spin tensor $S^{a b}$ by

$$
M \dot{\gamma}_{a}=P_{a}-n_{a b} P^{b} / M-\frac{S_{a}^{b}\left[q F_{b c}^{\mathrm{ext}}\left(P^{c}-n^{c}{ }_{d} P^{d} / M\right)+M f_{b}\right]}{M^{2}-\frac{1}{2} q S^{c d} F_{c d}^{\mathrm{ext}}} .
$$

The evolution equations for $P^{a}$ and $S^{a b}$ are

$$
\begin{gathered}
\dot{P}_{a}=q F_{a b}^{\mathrm{ext}} \dot{\gamma}^{b}+f_{a} \\
\dot{S}_{a b}=2 P_{[a} \dot{\gamma}_{b]}+n_{a b} .
\end{gathered}
$$

These equations are to be supplemented by $P_{a} S^{a b}=0$, which, if imposed as an initial 
condition, can be shown to be preserved under evolution (see [16]). At the level of what is known purely from lowest order perturbation theory as derived in section IV, there is no reason to prefer the system (144)-(150) to the system (140)-(142). However, in cases where the self-force term in eq. (144) is negligible, the system (144)-(150) yields an exact conservation law when there is a Killing field that Lie derives $F_{a b}^{\text {ext }}$-a feature that would hold exactly for test body motion. Therefore, it appears that in this respect the system (144) $-(\underline{150})$ is superior to the system (140)-(142).

\section{SUMMARY}

We have given a rigorous and systematic treatment of particle motion in classical electromagnetism. We considered a one-parameter-family of solutions to the Maxwell and matter equations (11)-(3) and (7) containing a body that "shrinks down" to zero size, mass, and charge according to the scaling assumptions of section II. We found that the lowest-order description of the body is that of a point particle (29) and (46) moving according to the Lorentz force law (48). The first-order corrections to this motion-including self-force, dipole force, and spin force effects - are then given by our rigorous perturbative result (134)-(136)). Finally, we addressed the issue of finding a self-consistent perturbative equation associated with our perturbative result. We argued that the naive self-consistent perturbative equations (140) -(142) can be modified by "reduction of order" to provide appropriate self-consistent perturbative equations. In the case of negligible spin and electromagnetic dipole moment, this reduces to the reduced-order ALD equation.

Acknowledgements This research was supported in part by NSF grants PHY04-56619 and PHY08-54807 to the University of Chicago.

\section{APPENDIX A: SCALING PROPERTIES OF THE SELF-FIELD}

This appendix derives the two properties of $F_{\mu \nu}^{\text {self }}\left(\lambda, t, x^{i}\right)$ that were claimed to hold at the end of section II for families of current distributions satisfying eq. (19).

We first show that the retarded field $F_{\mu \nu}^{\text {self }}\left(\lambda, t, x^{i}\right)$ satisfies the scaling relation, eq. (20). In global inertial coordinates $\left(t, x^{i}\right)$, the Lorenz-gauge vector potential for the retarded solution 
with source $J^{\mu}$ is given by

$$
A_{\mu}^{\text {self }}\left(\lambda, t, x^{i}\right)=\int \mathrm{d}^{3} x^{\prime}\left[\frac{J_{\mu}\left(\lambda, t-\left|x^{i}-x^{\prime i}\right|, x^{\prime j}\right)}{\left|x^{i}-x^{\prime i}\right|}\right],
$$

where, in our case, we have $J^{\mu}\left(\lambda, t, x^{i}\right)=\lambda^{-2} \tilde{J}^{\mu}\left(\lambda, t,\left[x^{i}-z^{i}(t)\right] / \lambda\right)$ with $\tilde{J}^{\mu}$ smooth in all variables and of compact support in the spatial variables. Now introduce scaled coordinates $\bar{x}^{i}=\left[x^{i}-z^{i}(t)\right] / \lambda$ and $\bar{x}^{i}=\left[x^{i}-z^{i}(t)\right] / \lambda$. Using these definitions together with eq. (19) and the coordinate transformation $\bar{y}^{i}=\bar{x}^{\prime i}-\bar{x}^{i}$, it is easily seen that

$$
A_{\mu}^{\text {self }}\left(\lambda, t, x^{i}\right)=\int \mathrm{d}^{3} \bar{y}\left[\frac{\tilde{J}_{\mu}\left(\lambda, t-\lambda\left|\bar{y}^{i}\right|, \bar{x}^{j}+\bar{y}^{j}+\left[z^{j}(t)-z^{j}\left(t-\lambda\left|\bar{y}^{k}\right|\right)\right] / \lambda\right)}{\left|\bar{y}^{i}\right|}\right] .
$$

Since $z^{i}(t)$ is smooth, there must exist a smooth function $V^{i}$ such that

$$
z^{i}(t)-z^{i}\left(t-\lambda\left|\bar{y}^{j}\right|\right)=\lambda\left|\bar{y}^{j}\right| V^{i}\left(t, \lambda\left|\bar{y}^{j}\right|\right)
$$

For later reference, we note that since $x^{i}=z^{i}(t)$ defines a timelike curve, it follows from the mean value theorem that $\left|V^{i}\right|<1$. Substituting in eq. (A2), we see that the integrand is smooth in $\left(\lambda, t, \bar{x}^{i}\right)$ and that the convergence properties of the $\bar{y}$-integral are sufficient to allow us to interchange differentiation with respect to $\left(\lambda, t, \bar{x}^{i}\right)$ with integration. It follows that in global inertial coordinates, $A_{\mu}^{\text {self }}$ smooth in these variables, i.e., $A_{\mu}^{\text {self }}$ is of the form

$$
A_{\mu}^{\text {self }}\left(\lambda, t, x^{i}\right)=\tilde{A}_{\mu}\left(\lambda, t,\left[x^{i}-z^{i}(t)\right] / \lambda\right) .
$$

where $\tilde{A}_{\mu}\left(\lambda, t, \bar{x}^{i}\right)$ is smooth in all of its arguments. Differentiating with respect to the spacetime variables, we find that $F_{\mu \nu}^{\text {self }}\left(\lambda, t, x^{i}\right)$ takes the form (20) in global inertial coordinates. By the same type of argument as given in sect. III, it then follows that $F_{\mu \nu}^{\text {self }}\left(\lambda, t, x^{i}\right)$ takes the general form (20) in arbitrary coordinates for which $\nabla_{\mu} t$ is timelike, as we desired to show.

We now derive a certain falloff behavior for $F_{\mu \nu}^{\text {self }}$ outside of the body, specifically that, in terms of the parameters

$$
\alpha \equiv\left|x^{i}-z^{i}(t)\right|, \quad \beta \equiv \lambda / \alpha,
$$


we have

$$
\lambda F_{\mu \nu}^{\mathrm{self}}=\beta^{2} \mathcal{F}_{\mu \nu}\left(t, \alpha, \beta, n^{i}\right)
$$

at least for $\alpha$ and $\beta$ in a sufficiently small neighborhood of $(0,0)$, where $\mathcal{F}_{\mu \nu}$ is smooth in all of its arguments and

$$
n^{i} \equiv\left[x^{i}-z^{i}(t)\right] / \alpha
$$

so that $\left|n^{i}\right|=1$. An analogous statement applied to one-parameter families of metrics was used in [9] in the analysis of gravitational self-force; as explained further in [9], this corresponds to what is needed to justify the use of matched asymptotic expansions. Matter distributions (if any) were not explicitly considered in [9], so the analogous behavior of metrics in that reference was an assumption. Here, we prove that the electromagnetic analog follows from a very natural form for the source, namely eq. (19).

We start, again, with eq. (A1) for the Lorentz-gauge vector potential $A_{\mu}^{\text {self }}\left(\lambda, t, x^{i}\right)$ in global inertial coordinates. Initially, we assume that $\alpha>0$, so that $\beta \geq 0$ and $n^{i}$ are well defined. We define

$$
w^{i}=x^{i}-z^{i}\left(t-\left|x^{j}-x^{\prime j}\right|\right)
$$

and we use eq. (A3) to write

$$
z^{i}\left(t-\left|x^{j}-x^{\prime j}\right|\right)=z^{i}(t)-\left|x^{j}-x^{\prime j}\right| V^{i}\left(t,\left|x^{j}-x^{\prime j}\right|\right)
$$

We also write

$$
w^{i}=\lambda \bar{w}^{i}=\alpha \beta \bar{w}^{i}
$$

The integrand in eq. (A1) is proportional to $\tilde{J}_{\mu}\left(\lambda, t-\left|x^{i}-x^{i}\right|, \bar{w}^{j}\right)$. Since $\tilde{J}_{\mu}$ is of compact support in the last argument, there exists a positive constant $D$ such that only points satisfying $\left|\bar{w}^{i}\right|<D$ contribute to the integral. Consequently, we may assume that $\left|\bar{w}^{i}\right|<D$ in the following.

Equations (A8)-(A10) together with the above definitions of $\alpha$ and $n^{i}$ yield

$$
x^{i}-x^{i}=\alpha\left(n^{i}-\beta \bar{w}^{i}\right)+\left|x^{j}-x^{\prime j}\right| V^{i}\left(t,\left|x^{k}-x^{\prime k}\right|\right) .
$$


Thus, taking the squared magnitude of both sides of this equation, we obtain

$$
y^{2}=\left|\alpha\left(n^{i}-\beta \bar{w}^{i}\right)+y V^{i}(t, y)\right|^{2}
$$

where we have written

$$
y \equiv\left|x^{i}-x^{i}\right|
$$

Treating eq. (A12) as though it were a quadratic equation in $y$ (even though $V^{i}$ depends on $y$ ), one finds that

$$
y=\frac{\alpha\left|n^{i}-\beta \bar{w}^{i}\right|}{1-\left|V^{j}\right|^{2}}\left(\left|V^{k}\right| \cos \theta+\sqrt{1-\left|V^{k}\right|^{2}+\left|V^{k}\right|^{2} \cos ^{2} \theta}\right)
$$

where $\cos \theta$ is defined by

$$
\left|V^{k}\right| \cos \theta \equiv \frac{V_{i}\left(n^{i}-\beta \bar{w}^{i}\right)}{\left|n^{j}-\beta \bar{w}^{j}\right|}
$$

and $V^{i}$ is evaluated at $(t, y)$. Since $y$ appears on both the left and right sides, eq. (A14) is not a solution for $y$ but rather is a relation that must be satisfied by the variables $y, t, \alpha$, $\beta, n^{i}$, and $\bar{w}^{i}$.

As already noted below eq. (A33), we have $\left|V^{i}\right|<1$. Furthermore, since $\left|\bar{w}^{i}\right|<D$, if we restrict to $\beta<1 / D$, then $\left|n^{i}-\beta \bar{w}^{i}\right|>0$. Consequently, for $\beta<1 / D$, the right side of eq. (A14) is of the form of $\alpha$ times a smooth, nonvanishing function of $\left(t, y, n^{i}, \beta \bar{w}^{i}\right)$. We may therefore solve this equation for $\alpha$, obtaining a solution of the form

$$
\alpha=y H\left(t, y, n^{i}, \beta \bar{w}^{i}\right)
$$

where $H$ is a smooth, nonvanishing function of all of its arguments.

Thus far, we have assumed that $\alpha>0$, which implies $y>0$ given our restrictions that $\left|\bar{w}^{i}\right|<D$ and $\beta<1 / D$. However, eq. (A14) makes perfectly good mathematical sense - and $H$ in eq. (A16) remains smooth - if we extend the domain of $y$ and $\alpha$ to include 0. (Indeed, eq. (A14) makes sense - and $H$ remains smooth - even for (unphysical) negative values of $y$, $\beta$, and $\alpha$.) We may therefore apply the inverse function theorem at $y=\alpha=0$ to conclude that in a sufficiently small neighborhood of $\alpha=0, y$ can be written as

$$
y=\alpha L\left(t, \alpha, n^{i}, \beta \bar{w}^{j}\right)
$$


where $L$ is a smooth, positive function of its arguments.

Rearranging (A8), we have

$$
x^{i}=\alpha \beta \bar{w}^{i}+z^{i}\left(t-\alpha L\left(t, \alpha, n^{j}, \beta \bar{w}^{k}\right)\right) .
$$

We now return to eq. (A1) and make the coordinate transformation $x^{i} \rightarrow \bar{w}^{i}$. Using eq. (A18), we see that the Jacobian of this coordinate transformation takes the form

$$
\left|\frac{\partial x^{\prime i}}{\partial \bar{w}^{j}}\right|=(\alpha \beta)^{3} W\left(t, \alpha, \beta, n^{i}, \bar{w}^{j}\right)
$$

where $W$ is a smooth function of its arguments. Substitution into (A1) finally shows that

$$
A_{\mu}^{\text {self }}\left(\lambda, t, x^{i}\right)=\beta \int \mathrm{d}^{3} \bar{w}\left(\frac{\tilde{J}_{\mu}\left(\alpha \beta, t-\alpha L\left(t, \alpha, n^{i}, \beta \bar{w}^{j}\right), \bar{w}^{k}\right)}{L\left(t, \alpha, n^{i}, \beta \bar{w}^{j}\right)}\right) W\left(t, \alpha, \beta, n^{l}, \bar{w}^{h}\right)
$$

The denominator here has been shown to be strictly positive over the range of $\bar{w}^{i}$ for which the numerator is non-vanishing. Both $L$ and $W$ are smooth and the integral is being taken over a compact region. The integral is therefore smooth in $t, \alpha, \beta$, and $n^{i}$, i.e., there exists a smooth $\mathcal{A}_{\mu}\left(t, \alpha, \beta, n^{i}\right)$ satisfying

$$
A_{\mu}^{\text {self }}=\beta \mathcal{A}_{\mu}
$$

for sufficiently small $\alpha$ and $\beta$. Differentiating to obtain the field strength shows that

$$
F_{\mu \nu}^{\text {self }}=(\beta / \alpha) \mathcal{F}_{\mu \nu}\left(t, \alpha, \beta, n^{i}\right)
$$

for some $\mathcal{F}_{\mu \nu}$ smooth in all of its arguments. Since $\lambda=\alpha \beta$, it follows immediately that $\lambda F_{\mu \nu}^{\text {self }}$ satisfies eq. (参). We have derived eq. (A6) in global inertial coordinates, but it is readily seen that this form is preserved under an arbitrary smooth transformation to new coordinates for which $\nabla_{\mu} t$ is timelike.

\section{APPENDIX B: "ELECTRON" MOTION}

It has been stressed in subsection IVC that the perturbed equations of motion that can be derived using only Maxwell's equations and conservation of total stress-energy do not 
constrain the time evolution of the electromagnetic dipole moment $Q_{a b}$. Thus, in order to have a deterministic system, the equations we derived in subsection IV C would have to be supplemented by an evolution law for $Q_{a b}$ arising from the particular matter model under consideration. A particularly simple and interesting case is to assume that for some (time independent) constant $C$ we have

$$
Q_{a b}=-C S_{a b}
$$

From eqs. (14) and (15) together with our center of mass condition $u^{a} S_{a b}=0$, it is easily seen that this is equivalent to having a vanishing electric dipole moment of the body and a magnetic moment proportional to its spin, i.e.,

$$
p_{a}=0, \quad \mu_{a}=C S_{a} .
$$

Since we have derived an evolution equation for $S_{a b}$, eq. (B1) serves to fix the time evolution of $Q_{a b}$ as well. As discussed at the beginning of section V, eqs (134)-(136) together with eq. (B1) and $u^{a} S_{a b}=0$ comprise a well-posed deterministic system for determining the perturbed motion. Note that since the electric dipole moment vanishes, we have $\delta \hat{m}=\delta m$ (see eq. (131)).

Since many elementary particles satisfy eq. (B1), we may view the self-consistent perturbative system eqs. (140)-(142) together with eq. (B1) as providing a simple, classical model for the motion of an elementary particle, such as an electron. To write these equations in a more familiar form, it is useful to decompose $F_{a b}^{\text {ext }}$ into rest-frame electric and magnetic fields via

$$
F_{a b}^{\mathrm{ext}}=2 u_{[a} E_{b]}+\epsilon_{a b c d} u^{c} B^{d}
$$

The evolution equation (141) for the angular momentum can then be written as

$$
\frac{\mathrm{D}}{\mathrm{d} \tau} S_{a}=-\epsilon_{a b c d} u^{b} \mu^{c} B^{d}+2 u_{[a} S_{b]} a^{b}
$$

Since $\mu_{a}=C S_{a}$, the right side is orthogonal to $S_{a}$, so

$$
\frac{\mathrm{d}}{\mathrm{d} \tau}\left(S_{a} S^{a}\right)=0
$$

i.e., the spin vector does not change its magnitude under time evolution. Clearly, $\mu_{a}$ also 
does not change its magnitude, i.e., the body has a "permanent" magnetic dipole moment.

The evolution equation (142) for mass reduces to

$$
\frac{\mathrm{d}}{\mathrm{d} \tau}\left(m+\mu_{a} B^{a}\right)=0
$$

where we have dropped the "hat" on $m$ since there is no distinction between $\hat{m}$ and $m$ when the electric dipole moment vanishes. Although $m$ does not remain constant, it is clear that we may define a new quantity

$$
m_{*} \equiv m+\mu_{a} B^{a}
$$

that is conserved. Note that the standard expression for interaction energy, $-\mu_{a} B^{a}$, is being subtracted from $m$ in this equation-rather than added to $m$ - to define $m_{*}$.

Note that the failure of the "rest mass" $m$ to be constant resolves a paradox concerning what one is taught in elementary physics courses: On one hand, one is (correctly) taught that an external magnetic field can "do no work" on a body, so a body moving in an external magnetic field cannot gain energy. On the other hand, one is (also correctly) taught that a magnetic dipole released in a non-uniform external magnetic field will gain kinetic energy. Where does this kinetic energy come from? Equation (B6]) shows that it comes from the rest mass of the body. ${ }^{14}$

We now consider the motion of the body, as described by eq. (140). Using eq. (B1), we obtain

$$
\begin{aligned}
m a_{a}=q E_{a} & +\left(\delta_{a}^{b}+u_{a} u^{b}\right)\left(\frac{2}{3} q^{2} \frac{\mathrm{D} a_{b}}{\mathrm{~d} \tau}+\frac{1}{2} \epsilon^{c d e f} u_{c} \mu_{d} \nabla_{b} F_{e f}^{\text {ext }}\right) \\
& +\epsilon_{a b c d} u^{b} S^{c} \frac{\mathrm{D}}{\mathrm{d} \tau}\left(C E^{d}-a^{d}\right)+2 \mu_{[a} B_{b]}\left(C E^{b}-a^{b}\right)
\end{aligned}
$$

where the terms involving acceleration on the right side are understood to be eliminated by reduction of order. ${ }^{15}$ If $q \neq 0$, it is conventional to write $C$ in terms of a dimensionless

\footnotetext{
${ }^{14}$ By contrast, for a "permanent electric dipole", i.e., $Q_{a b}=2 u_{[a} p_{b]}$ with $D_{F} Q_{a b} / d \tau=0$, it follows from eq. (142) that the original mass $m=\hat{m}+p_{a} E^{a}$ is conserved. The kinetic energy gained by an electric dipole released in a non-uniform electric field comes from the work done on the body by the electric field.

${ }^{15}$ In particular, a term proportional to $\epsilon_{b c d f} a^{c} \mu^{d} E^{f}$ has been set to zero since $a^{a}$ is to be replaced by $q E^{a} / m$.
} 
$g$-factor defined by $C=q g / 2 m$, in which case

$$
C E^{a}-a^{a}=\frac{(g-2) q}{2 m} E^{a}
$$

where we have again used $a^{a}=q E^{a} / m$ for terms on the right side. In the case of an electron or muon, $g$ is very nearly 2 , so the last two terms on the right side of eq. (B8 $)$ should be negligible. However, $g$ can be very different from 2 for composite particles, in which case the last two terms in eq. (B8) need not be negligible.

Another point worth noting with regard to both eq. (140) and eq. (B8) is that the Lorentz-Dirac self-force is not the only term involving $\mathrm{D} a_{b} / \mathrm{d} \tau$. A "jerk" force also occurs in conjunction with the spin of the body in the second line of eq. (B8). (Note that this force is orthogonal to the ALD force.) For a body of charge $q$ equal to the charge of the electron $e$, and with spin of order $\hbar$, the ratio of the magnitude of the "spin jerk" term to the ALD term is of order $(g-2) \hbar / e^{2}=(g-2) / \alpha$. Thus, if $g \neq 2$, the "spin jerk" term can dominate over the ALD term. However, the effects of the "spin jerk" term probably do not accumulate significantly over time.

It also interesting to ask when the ALD self-force becomes comparable to the Lorentz force. In this situation, it is likely that higher order corrections to the self-force will be large, and our perturbative equations are unlikely to be reliable. It is easily seen that for an external electromagnetic field that oscillates with frequency $\omega$, the condition that the ALD self-force is much smaller than the Lorentz force is simply

$$
q^{2} \omega \ll m
$$

For an electron, this reduces to

$$
\alpha \hbar \omega \ll m .
$$

It seems unlikely that a classical description of electron motion would be possible in any case if this condition were violated.

Another criterion for the validity of our perturbative equations is that the change in mass under time evolution - which is a perturbative effect - be small compared with the mass, i.e., that

$$
|\mu||B| \ll m
$$


If $g \sim 1$ and $|q|=e$, this reduces to

$$
|B| \ll \frac{m^{2}}{e \hbar} \sim\left(4 \times 10^{13} \mathrm{G}\right)\left(\frac{m}{500 \mathrm{keV}}\right)^{2}
$$

Magnetic fields expected to exist near some neutron stars are expected to violate this bound. It seems unlikely that any presently known classical equations of motion can adequately describe the behavior of electrons when this bound is violated.

[1] P. A. M. Dirac, Proc. R. Soc. A 167, 148 (1938)

[2] R. Geroch and J. Traschen, Phys. Rev. D 36, 1017 (1987)

[3] M. Abraham Ann. Physik 10, 105 (1903)

[4] H. A. Lorentz, Theory of Electrons (Dover, New York 1952 reprinted from 1915), 2nd ed.

[5] G. A. Schott, Proc. Roy. Soc. A159, 548 (1937); A159, 570 (1937)

[6] R. Geroch, arXiv:gr-qc/9602055 (1996)

[7] A. I. Harte, Phys. Rev. D 73, 065006 (2006)

[8] A. I. Harte, arXiv:0903.0167 (2009)

[9] S.E. Gralla and R.M. Wald, Class. Quantum Grav. 25205009 (2008)

[10] E. J. Moniz and D. H. Sharp, Phys. Rev. D 15, 2850 (1977)

[11] C. J. Eliezer, Proc. R. Soc. A 194, 543 (1948)

L. D. Landau and E.M. Lifshitz 1962 The Classical Theory of Fields (Oxford: Pergamon Press)

J. Z. Simon and L. Parker, Phys. Rev. D 47, 1339 (1993)

E. Flanagan and R.M. Wald, Phys. Rev. D 54, 6233 (1996)

F. Rohrlich, Phys. Rev. E 77, 046609 (2008)

[12] R. M. Wald 1984 General Relativity (Chicago, IL: University of Chicago Press)

[13] E. Poisson, Liv. Rev. Rel. 76 (2004)

[14] W. L. Burke, J. Math. Phys. (N.Y.) 12, 401 (1971)

P. D. D'Eath, Phys. Rev. D 11, 1387 (1975)

R. Kates, Phys. Rev. D 22, 1853 (1980)

K. S. Thorne and J. B. Hartle, Phys. Rev. D 31, 1815 (1985) 
Y. Mino, M. Sasaki, and T. Tanaka, Phys. Rev. D 55, 3457-3476, (1997)

[15] S.E. Gralla, A.I. Harte, and R.M. Wald, in preparation

[16] J. Ehlers and E. Rudolph, Gen. Rel. Grav. 8, 197 (1977) 